\title{
Análisis de fracturas geológicas en el pozo Agrícola Oriental 2C, Ciudad de México y su relación con fallas mayores
}

\author{
Alberto Vásquez-Serrano ${ }^{1,2^{*}}$, Rebeca Camacho-Rangel ${ }^{3}$, \\ José Luis Arce-Saldaña ${ }^{1}$ y Eric Morales-Casique ${ }^{1}$
}

${ }^{1}$ Departamento de Procesos Litosféricos, Instituto de Geología, Universidad Nacional Autónoma de México, Circuito de la Investigación Científica s/ $n$, Ciudad Universitaria, Coyoacán, Ciudad de México, C.P. 04510, México.

${ }^{2}$ Centro de Geociencias, Universidad Nacional Autónoma de México, Blvd. Juriquilla 3001, Campus UNAM, Juriquilla La Mesa, Juriquilla, Querétaro, C.P. 76230, México.

${ }^{3}$ Colegio de Geofísica, Facultad de Ingeniería, Benemérita Universidad Autónoma de Puebla, 4 Sur No. 104, Centro Histórico, Ciudad de Puebla, Puebla, C.P. 72000, México.

*alberto-vasquez@ciencias.unam.mx

\section{RESUMEN}

En años recientes, en la Ciudad de México se han realizado varios pozos exploratorios de hasta $2000 \mathrm{~m}$ de profundidad, con el fin de encontrar acuíferos que ayuden a disminuir el desabasto de agua potable para el área metropolitana. En particular, en el pozo Agrícola Oriental 2C, perforado en la Alcaldía de Iztacalco, se recuperaron varios núcleos, uno de los cuales mide $9 \mathrm{~m}$, ubicado entre $1550 \mathrm{y}$ $1559 \mathrm{~m}$ de profundidad, cercano al contacto entre rocas calcáreas del Cretácico y rocas volcánicas del Oligoceno, a $\sim 1560 \mathrm{~m}$ de profundidad. El núcleo muestra que las rocas volcánicas se encuentran afectadas por una cantidad importante de fracturas, las cuales pueden ser divididas en dos familias con base en su relleno y relaciones de corte (F1 y F2). Las fracturas más jóvenes (F2) se relacionan con una falla, llamada informalmente en este trabajo falla Agrícola Oriental, que pone en contacto rocas sedimentarias y volcánicas.

Las fracturas fueron caracterizadas mediante un análisis detallado de la cantidad, geometría y distribución a lo largo de una línea de muestreo, tomando en cuenta ambas familias de fracturas. Estas variables fueron expresadas en términos de intensidad de fracturas, dimensión fractal de caja y correlación, exponente de Lyapunov, coeficiente de variación y exponente de frecuencia acumulada. Los resultados obtenidos muestran que el espaciamiento entre fracturas, así como la apertura, se ajustan muy bien a una ley de potencia en un gráfico bilogarítmico de su frecuencia acumulada, por lo que las fracturas exhiben un comportamiento fractal. El valor de la intensidad de fracturas, dimensión fractal, exponente de Lyapunov y exponente de frecuencia acumulada de la apertura, disminuyen progresivamente con el aumento de la distancia a la falla, mientras que el coeficiente de variación y exponente de frecuencia acumulada del espaciamiento presentan un patrón contrario. Estos comportamientos suelen ser típicos en una zona de cizalla común, sin embargo, un aspecto importante encontrado en este estudio es la alteración de estos patrones con la presencia de un grupo de fracturas preexistentes (F1) a la formación de un nuevo grupo de fracturas (F2), lo cual provoca un aumento en la cantidad de fracturas y una redistribución de estas estructuras dentro de la zona de falla. Adicionalmente, con base en las características de las fracturas estudiadas, se propone que la falla encontrada en este estudio pertenece al sector norte de la fosa de Santa Catarina y tiene una orientación ENE-WSW.

Palabras clave: fractura geológica; análisis fractal; arreglo de fracturas; intensidad de fracturas; falla Agrícola Oriental.

\section{ABSTRACT}

In the last years several exploratory wells have been drilled in Mexico City down to $2000 \mathrm{~m}$ depth, with the aim to explore deep aquifers to cover the water deficiency in the metropolitan area. In particular, in the Agricola Oriental 2C well, located in the Iztacalco municipality, several cores were recovered; one of them is $9 \mathrm{~m}$ long, located between 1550 and $1559 \mathrm{~m}$ depth. This core is situated close to the contact between an Oligocene volcanic unit and a Cretaceous limestone at $\sim 1560 \mathrm{~m}$ depth. The study core shows that the volcanic rocks are affected by an important number of fractures, which can be divided into two families according to their intersection relationships and fill material (F1 and F2). The younger fractures (F2) are related with a fault, informally named here as Agricola Oriental fault, which sets in tectonic contact the sedimentary and the volcanic rocks.

The fractures were characterized through a detailed analysis of their number, geometry and spatial arrangement, using the scan line method and taking into account both families of fractures. These variables were expressed such as fracture intensity, box and correlation fractal dimensions, Lyapunov exponent, variation coefficient, and cumulative frequency exponent. Our results show that the spacing between fractures and the aperture follow a power law in a log-log graph of the cumulative frequency analysis that results in a fractal behavior. The pattern values showed in the fracture intensity, fractal dimension, Lyapunov exponent, and aperture cumulative frequency exponent progressively diminish as the distance to the fault increases. In turn, the coefficients of variation and spacing cumulative frequency exponent have a different pattern: 
their values increase with the distance to the fault. These behaviors are typical of a common shear zone; however, in our analysis, the anomalous variation of the study parameters in the fault damaged zone is related to the presence of a previous fracture system (F1). These fractures induced an important increase in the amount of fractures and change their distribution in the damage zone. In addition, based on the fracture characteristics, we proposed that the fault found in the well is related to the northern limit of Santa Catarina graben and has an ENE-WSW orientation.

Key words: fracture; fractal analysis; fracture array; fracture intensity; Agrícola Oriental Fault.

\section{INTRODUCCIÓN}

Las fracturas son estructuras comunes en los medios geológicos, se forman bajo condiciones de deformación frágil y su estudio requiere de observaciones sistemáticas a través de metodologías particulares (Ramsay y Huber, 1987; Cladouhos y Marrett, 1996; Babadagli, 2001; Mauldon et al., 2001; Nieto-Samaniego et al., 2003, 2005; Gale et al., 2014; Hooker et al., 2018; Laubach et al., 2018). Mecánicamente, una fractura se define como el resultado de un proceso de deformación, el cual se manifiesta mediante la separación o fragmentación de un cuerpo sólido (roca) que está sujeto a un determinado estado de esfuerzos (Bahat et al., 2005). Las fracturas se generan por una concentración de esfuerzos en las rocas y se propagan a través de un gran número de heterogeneidades (Griffith, 1920). El análisis cuantitativo de las fracturas geológicas se ha desarrollado ampliamente en los últimos años, debido al papel que juegan en el estudio de fenómenos como estabilidad de taludes, hidrogeología y análisis de yacimientos que involucran flujo de fluidos (Babadagli, 2001; Laubach y Ward, 2006; Hooker et al., 2013; Gale et al., 2014). Dicho desarrollo ha sido acompañado con el diseño de nuevas metodologías para describir su geometría y distribución, tanto en superficie como en núcleos obtenidos de perforaciones (Mauldon et al., 2001; Laubach et al., 2018). Los análisis hechos en núcleos han sido fundamentales para conocer la manera cómo se relacionan dichas estructuras con la porosidad y permeabilidad de una roca a profundidad en un sitio determinado (Jafari y Babadagli, 2008, 2009, 2010; Li et al., 2018). Los estudios geológico-estructurales realizados en núcleos han sido principalmente en rocas sedimentarias con enfoque en los hidrocarburos.

En la Ciudad de México durante los últimos años se han perforado cuatro pozos, con profundidades entre 1570 y $2008 \mathrm{~m}$ (pozo San Lorenzo Tezonco en 2012, pozos Agrícola Oriental 2B y 2C en 2016 y pozo Santa Catarina 3A en 2017; ver Figura 1c) con el objetivo de encontrar rocas que pudieran tener un potencial hidrogeológico (Morales-Casique et al. 2014; Lezama-Campos et al., 2016; MoralesCasique et al. 2016; Morales-Casique et al. 2017). La perforación de estos pozos ha sido acompañada por diversos estudios sobre la estratigrafía profunda de la ciudad, mediante la recuperación de núcleos y muestras de canal, así como el análisis de registros geofísicos (Arce et al., 2013, 2015; Lezama-Campos et al., 2016). Aunque se ha evaluado el potencial hidrogeológico que tienen las unidades litológicas cortadas en las perforaciones, no se ha tomado en cuenta las estructuras geológicas que las afectan (Morales-Casique et al., 2014; Morales-Casique et al., 2015). Los datos obtenidos de estas perforaciones han complementado los estudios realizados por Petróleos Mexicanos (Pérez-Cruz, 1988) durante el siglo pasado y han contribuido en gran medida a entender la estratigrafía del subsuelo de la Ciudad de México. A pesar de los avances obtenidos en la descripción y análisis de las rocas cortadas por los pozos, no se ha realizado un estudio detallado de las fracturas meso- y microscópicas que afectan a dichas rocas. El estudio de las fracturas y fallas en el subsuelo de la Ciudad de México es fundamental no sólo para determinar su papel en el flujo de fluidos, sino también para conocer su geometría, distribución y actividad reciente.

Los pozos Agrícola Oriental 2B (19 $23^{\prime} 42.93^{\prime \prime} \mathrm{N}, 99^{\circ} 05^{\prime} 11.25^{\prime \prime} \mathrm{W}$; $2000 \mathrm{~m}$ de profundidad) y $2 \mathrm{C}\left(19^{\circ} 23^{\prime} 43.25^{\prime \prime} \mathrm{N}, 99^{\circ} 5^{\prime} 11.01^{\prime \prime} \mathrm{W} ; 1570 \mathrm{~m}\right.$ de profundidad) fueron perforados en el año 2016 en la Alcaldía de Iztacalco (Figura 2), a unos metros del pozo Mixhuca-1 (19²3'19”N, 9905'17”W), éste último perforado por PEMEX en 1985 (Pérez-Cruz, 1988). Al igual que el pozo Mixhuca-1, el Agrícola Oriental 2B también cortó el contacto entre las rocas calcáreas del Cretácico Tardío y volcánicas con una edad probablemente mayor a $29 \mathrm{Ma}$ (Pérez-Cruz, 1988), según lo observado en el pozo Mixhuca-1 (Figura 3). La profundidad a la cual se cortó dicho contacto fue de $\sim 1560 \mathrm{~m}$. Uno de los objetivos principales de las perforaciones, fue la recuperación de núcleos en el contacto entre rocas calcáreas y volcánicas, que permitiera observar las características de las rocas volcánicas cerca del contacto. En el pozo Agrícola Oriental 2C se recuperó el $100 \%$ del núcleo $\mathrm{N} 2 \mathrm{AO} 2 \mathrm{C}$, entre 1550 y $1559 \mathrm{~m}$ de profundidad, y que corta las rocas volcánicas cerca del contacto (reportes PEMEX-SACMEX, 2016a, 2016b). Desafortunadamente, en la parte calcárea no se obtuvieron núcleos próximos al contacto que permitieran ver el estado físico de éstas. A pesar de ello, se pudo observar que las rocas volcánicas localizadas cerca del contacto están afectadas de manera importante por una serie de fracturas y fallas. Esto ofrece una excelente oportunidad para conocer las características de estas estructuras en el subsuelo de la Ciudad de México.

En este trabajo se analizan las fracturas que afectan a las rocas volcánicas que se encuentran en contacto con las rocas calcáreas en el pozo Agrícola Oriental 2C (Figuras 2 y 3 ). A través de un estudio detallado y utilizando diferentes parámetros, se describe su distribución y la relación que guardan con fallas kilométricas definidas en la cuenca de México (Figura 1c) (Pérez-Cruz, 1988; García-Palomo et al., 2008; Arce et al., 2013). Los resultados presentados aquí pueden ser de utilidad para conocer la contribución de las fracturas en el proceso de flujo de fluidos a profundidad y su posible relación con acuíferos profundos.

\section{MARCO GEOLÓGICO}

\section{Antecedentes}

La Ciudad de México se ubica dentro de una cuenca rodeada por una serie de cadenas montañosas de origen volcánico (Sierra de Guadalupe al norte, Sierra Chichinautzin al sur, Sierra de las Cruces al oeste y Sierra Nevada al este), las cuales pertenecen a la Faja Volcánica Transmexicana (FVTM, Figura 1). Dicha faja es un arco volcánico continental que se extiende por $\sim 1000 \mathrm{~km}$ de oeste a este en el centro de México y tiene una edad del Mioceno al Cuaternario (García-Palomo et al., 2000; Gómez-Tuena y Carrasco-Núñez, 2000; Ferrari et al., 2011). El arco volcánico está relacionado a un proceso de subducción de las placas de Cocos y Rivera bajo la placa Norteamericana (Figura 1a). Adicionalmente, su oblicuidad con respecto a la trinchera es una característica particular que lo diferencia de otros arcos volcánicos, la cual está relacionada con variaciones en la velocidad y ángulo de subducción (Molnar y Sykes, 1969; Stoiber y Carr, 1973; Pardo y Suárez 1993, 1995; Ferrari et al., 1999; Ferrari et al., 2011; Manea et al., 2013).

Las rocas que se encuentran aflorando dentro de la cuenca de México han sido descritas desde mediados del siglo XX por Fries (1956, $1960)$ y Mooser $(1963,1975)$ principalmente (Figura 1c). Dichos autores asignaron nombres formales a la mayoría de las rocas, los cuales se siguen utilizando en la actualidad. Un evento importante que marcó un parteaguas en el estudio de las rocas de la cuenca de México fue 


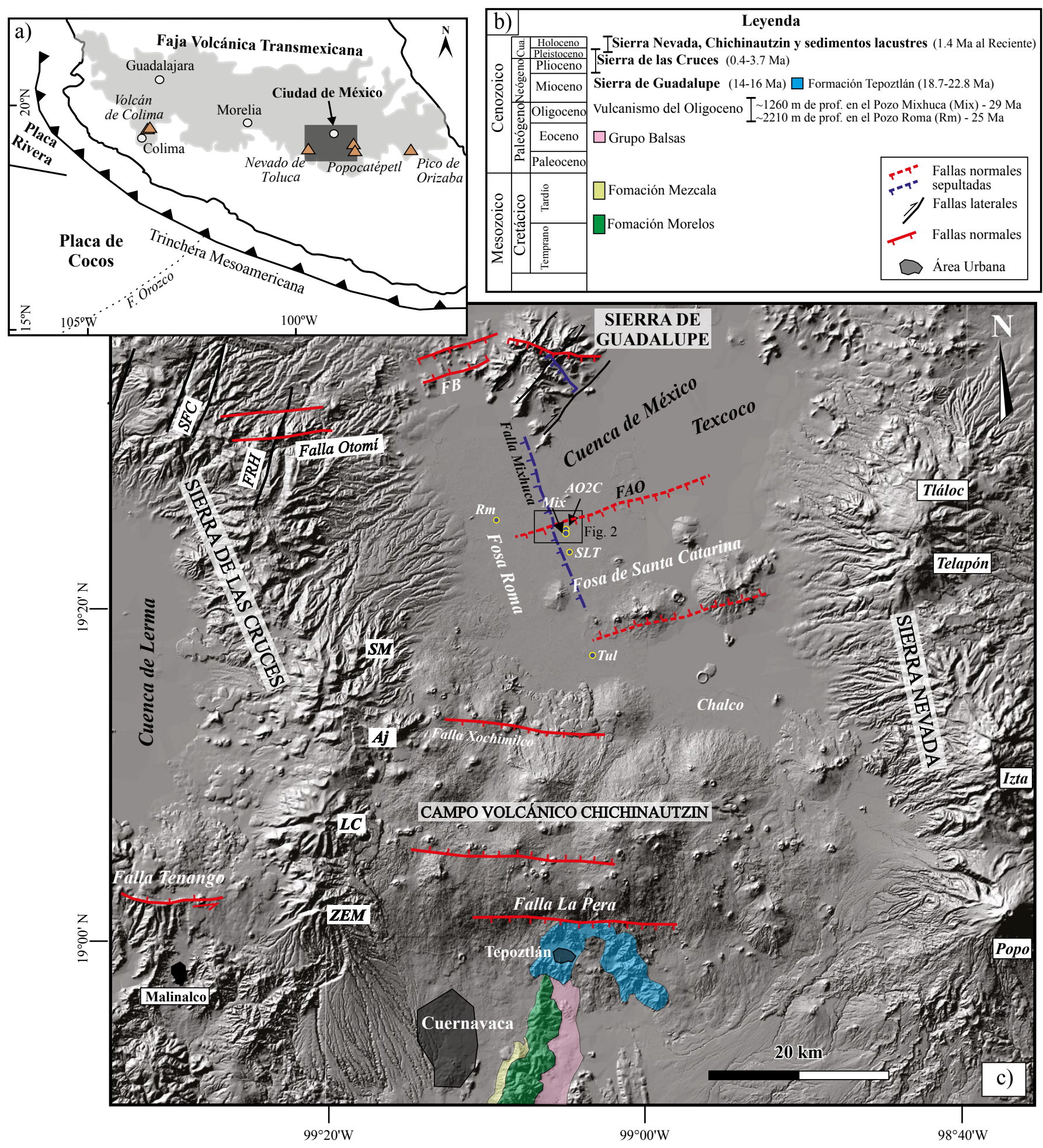

Figura 1. a) Mapa tectónico del centro-sur de México donde se muestra a la Faja Volcánica Transmexicana y la ubicación de la cuenca de México (Modificado de Arce et al., 2013). b) Columna estratigráfica representativa de la cuenca de México. c) Modelo digital de elevación donde se aprecian las sierras volcánicas que rodean a la cuenca de México, las principales fallas geológicas reportadas en trabajos previos (Alaniz-Álvarez et al., 1998; García-Palomo et al., 2008; Arce et al., 2013; Lermo et al., 2016) y los afloramientos de las rocas pre-oligocénicas al sur de la cuenca (Fries, 1956, 1960; González-Torres et al., 2015). Así mismo, se incluye la

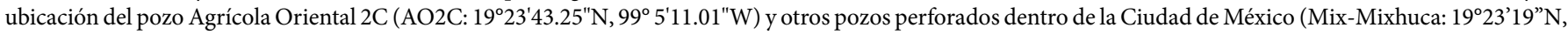

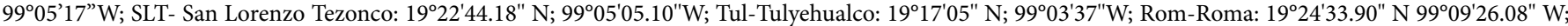
SC-Santa Catarina 3A: 19²17'45.84"N, 99 4'55.64"W). Las estructuras volcánicas son: SM-San Miguel, AJ-Ajusco, LC-La Corona, ZEM-Zempoala, Izta-Iztaccíhuatl, Popo-Popocatépetl (Arce et al., 2018; Macías et al., 2012) y estructuras tectónicas: FB-Fosa Barrientos, SFC-Sistema de Fallas Catedral, FRH-Falla Río Hondo (GarcíaPalomo et al., 2008), FAO-Falla Agrícola Oriental (en este trabajo). Se indican las fuentes de las edades de las siguientes formaciones o rasgos topográficos: Lenhardt et al. (2010) para la Fm. Tepoztlán; Lozano-Barraza (1968) para la Sierra de Guadalupe y la compilación hecha por Arce et al. (2008) para la Sierra de las Cruces. 


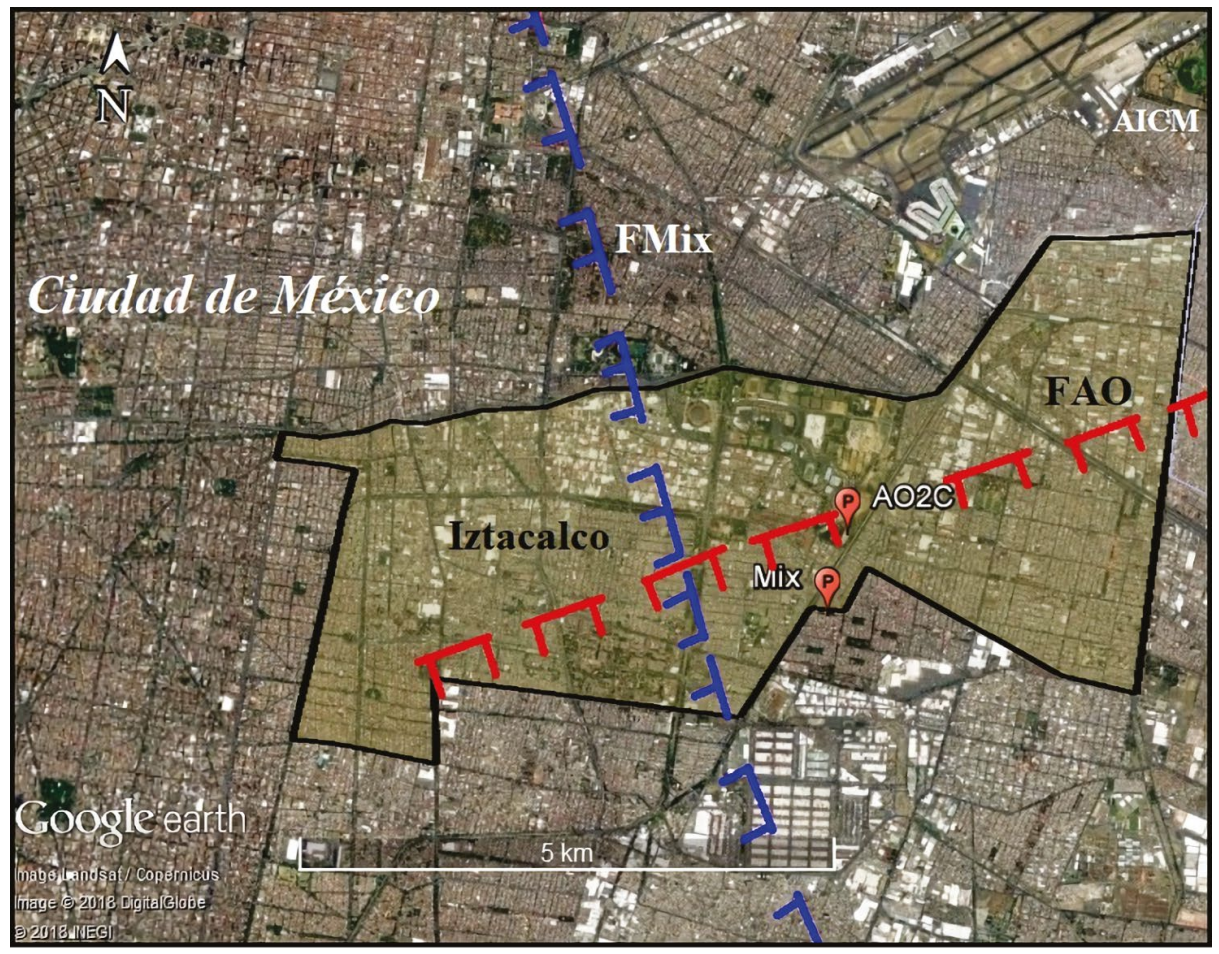

Figura 2. Localización de los pozos Mixhuca (Mix) y Agrícola Oriental 2C (AO2C) dentro de la alcaldía de Iztalcalco, al sur del Aeropuerto Internacional de la Ciudad de México (AICM). En el mapa también se aprecia la traza de las fallas Mixhuca (FMix) y Agrícola Oriental (FAO).

el sismo de 1985, debido a que surgió la necesidad de conocer con detalle la estratigrafía del subsuelo de la Ciudad de México. Por esta razón, se perforaron varios pozos profundos y se describió con cierto detalle la litología profunda (Pérez-Cruz, 1988; Pérez-Cruz, 1988). Adicionalmente, se han realizado estudios geológicos en las sierras que rodean la cuenca, proporcionando información valiosa sobre la edad, distribución y tipo de rocas que constituyen dichas sierras (Osete et al., 2000; Arce et al., 2008; García-Palomo et al., 2008; Cadoux et al., 2011; Macías et al., 2012). Así mismo, se han realizado estudios detallados de los sedimentos lacustres del Holoceno y Pleistoceno Tardío, aportando datos valiosos a la estratigrafía del Cuaternario y generando información paleoambiental (Lozano-García et al., 1993; Lozano-García y Ortega-Guerrero, 1998; Brown et al., 2012).

Recientemente, se han realizado estudios geocronológicos detallados de las muestras de canal y núcleos recuperados de los pozos perforados en la Ciudad de México. Dichos estudios han mostrado no sólo las variaciones litológicas en el subsuelo, sino también la edad de las rocas, con lo cual se han establecido correlaciones estratigráficas con algunas formaciones geológicas reportadas en sitios cercanos a la cuenca de México (Arce et al., 2013, 2015). En el caso del pozo San Lorenzo Tezonco (19²2'44.18" N; 99 $\left.05^{\prime} 05.10^{\prime \prime} \mathrm{W}\right)$, Arce et al. (2013) pudieron describir las variaciones litológicas y fechar las rocas encontradas a $\sim 1500 \mathrm{~m}$ en $18.23 \pm 0.17 \mathrm{Ma}$ (edad ${ }^{40} \mathrm{Ar}{ }^{139} \mathrm{Ar}$ en roca total). Una aportación importante realizada por estos autores fue la definición de una fosa con una orientación ENE-WSW, para explicar las variaciones en profundidad del contacto entre las rocas calcáreas del Cretácico Tardío y las rocas volcánicas que las cubren (Eoceno-Oligoceno), así como la presencia del alineamiento de volcanes monogenéticos de la Sierra de Santa Catarina. Finalmente, en el mismo pozo (San Lorenzo Tezonco), Arce et al. (2015) fecharon las rocas volcánicas más profundas cortadas por el pozo asignándoles una edad ${ }^{40} \mathrm{Ar}^{139} \mathrm{Ar}$ en roca total de $20.1 \pm 0.3 \mathrm{Ma}$.

\section{Estratigrafía en el centro de la cuenca de México}

Se han realizado varios trabajos geológicos en los alrededores de la cuenca de México con el objetivo de establecer su estratigrafía (Mooser, 1963, 1975; Bloomfield, 1975; Wallace y Carmichael, 1999; Márquez et al., 1999; Siebe, 2000; García-Palomo et al., 2006; Arce et al., 2008; Macías et al., 2012; Arce et al., 2015). Estos trabajos se han complementado con la información obtenida de los pozos profundos realizados en la Ciudad de México (Pérez-Cruz, 1988; Arce et al., 2013, 2015). Con base en los pozos, se ha podido establecer que existe un basamento calcáreo que se correlaciona con las formaciones geológicas del Cretácico que afloran al sur de la cuenca, en el área de Cuernavaca-Tepoztlán (Fries, 1960, Figuras 1b y 3a; Pérez-Cruz, 1988). Cubriendo al basamento se encuentran conglomerados calcáreos (Eoceno) pertenecientes al Grupo Balsas (González-Torres et al., 2015) y rocas volcánicas del Eoceno-Oligoceno (Pérez-Cruz, 1988). La columna continúa con depósitos volcánicos del Mioceno expuestos en la Sierra de Guadalupe (García-Palomo et al., 2006) y en los alrededores de Tepoztlán, Morelos (Lenhardt et al., 2010) (Figura 1b), asociadas con la actividad de la FVTM. Rocas de esta misma edad han sido cortadas en los pozos Mixhuca, Tulyehualco, San Lorenzo Tezonco y Roma (Pérez-Cruz, 1988; Arce et al., 2013, 2015). Cubriendo a las rocas miocénicas se encuentran depósitos volcánicos del Plioceno al Holoceno que también están relacionados con la FVTM (Figura 1b), los cuales afloran en la Sierra de las Cruces (Arce et al., 2008), Sierra Nevada (Macías et al., 2012) y Sierra Chichinautzin (Bloomfield, 1975; Wallace y Carmichael 1999; Márquez et al., 1999; Siebe, 2000; Siebe et al., 2005; Guilbaud et al., 2009, Agustín-Flores et al., 2011; Arce et al., 2015). Finalmente, en la cima de la columna existen sedimentos lacustres del Pleistoceno al Holoceno (Lozano-García et al., 1993, Figura 1b; Lozano-García y Ortega-Guerrero, 1998; Brown et al., 2012; Lozano-García et al., 2017).

Por otro lado, en el pozo Mixhuca, Pérez-Cruz (1988) divide la 
a) Mix $^{1}$-AO2C

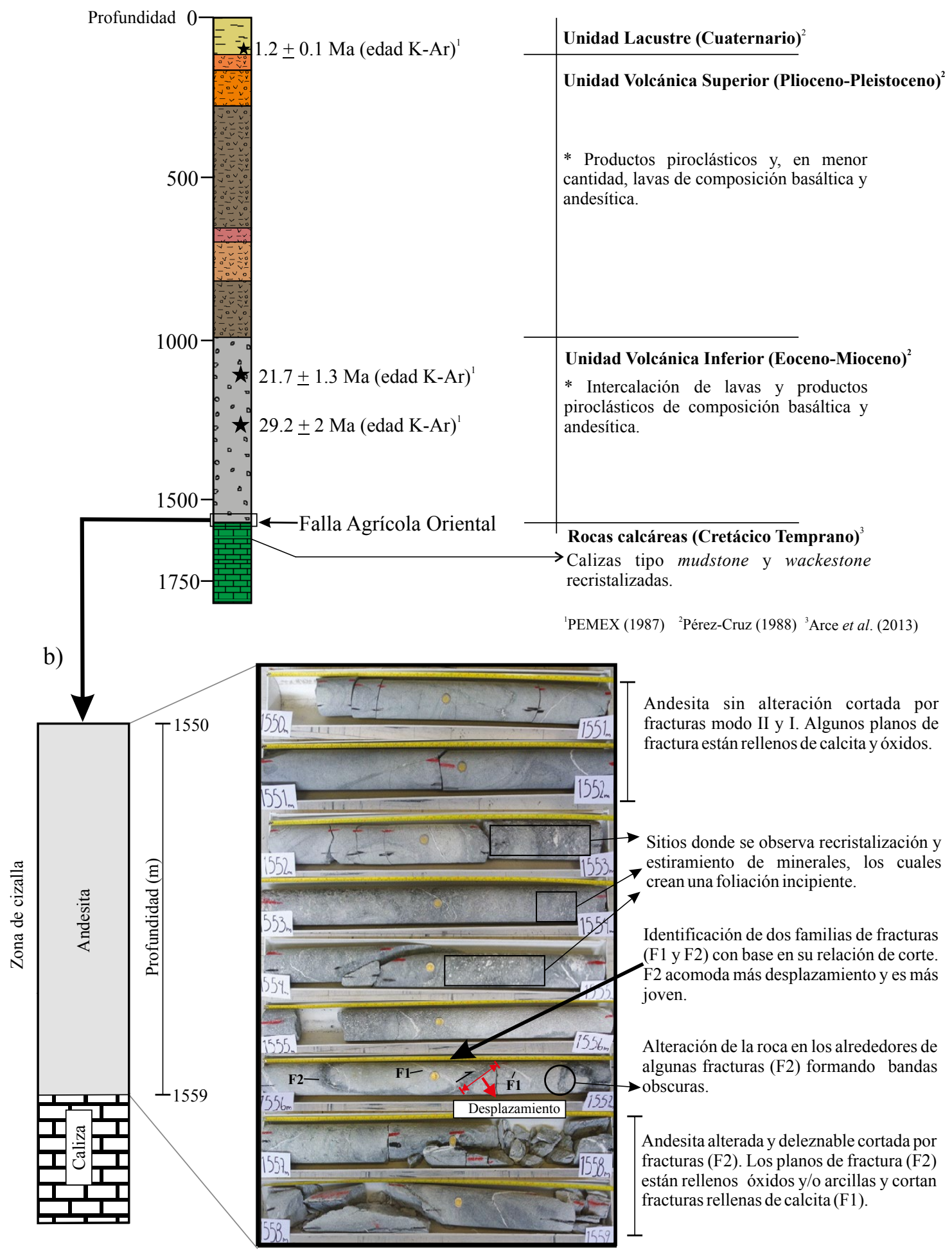

Figura 3. a) Columna estratigráfica representativa de los pozos Mixhuca y Agrícola Oriental 2C (AO2C) con la definición de cuatro unidades sugeridas por PérezCruz (1988). Adicionalmente se agregan las edades K-Ar en roca total reportadas por PEMEX (Pérez-Cruz, 1988), así como la ubicación del núcleo recuperado en el pozo $\mathrm{AO} 2 \mathrm{C}$ donde se localiza la zona de cizalla de la falla Agrícola Oriental. b) Estado de la andesita del núcleo recuperado, donde se observan las fracturas y el grado de alteración. Es importante notar la presencia de dos familias de fracturas que afectan a la roca en estudio: F1 (rellena de calcita) cortada por F2 (abiertas o rellenas de óxidos). Estas últimas generadas por la falla Agrícola Oriental que pone en contacto a rocas volcánicas probablemente del Oligoceno con rocas calcáreas del Cretácico (Pérez-Cruz, 1988). 
columna del pozo en cuatro unidades (Figura 3a), que desde la base son: a) Basamento calcáreo del Cretácico; b) Unidad Volcánica Inferior (UVI), c) Unidad Volcánica Superior (UVS) relacionada con la actividad de la FVTM y d) Unidad de depósitos lacustres y fluviales. Para el caso del pozo $\mathrm{AO} 2 \mathrm{C}$, no se tiene aún una columna definida, ya que no se cuenta con la edad de las rocas cortadas. Sin embargo, al ubicarse el pozo $\mathrm{AO} 2 \mathrm{C}$ muy cerca del pozo Mixhuca (Figura 2), se puede tomar la columna de este último como representativa del pozo AO2C (Figura 3a). De acuerdo con lo antes mencionado, es muy probable que las rocas del núcleo estudiado pertenezcan a la UVI y de edad Oligoceno (Figuras $1 \mathrm{~b}$ y $3 \mathrm{a}$ ).

\section{Sistemas de fallas en la cuenca de México}

En el centro de México, dentro de la FVTM, se han documentado por lo menos tres sistemas de fallas de tipo normal: NNW-SSE, E-W y NE-SW (Figura 1c) (Alaniz-Álvarez et al., 1998; García-Palomo et al., 2008). Algunos de estos sistemas han sido reactivados durante la fase de extensión cortical (N-S) observada actualmente dentro de la FVTM, heredados de eventos de extensión más antiguos (Alaniz-Álvarez, 2001, 2002). Estos tres sistemas de fallas están presentes en la mayor parte de la FVTM, siendo el sistema NNW-SSE el más antiguo (OligocenoMioceno) y el $\sim \mathrm{E}-\mathrm{W}$ el que actualmente se encuentra activo, acomodando un mayor desplazamiento (Alaniz-Álvarez et al., 1998).

En la zona occidental y noroccidental de la cuenca de México se han realizado algunos estudios de las fallas que afectan a las rocas de la Sierra de la Cruces y de la parte occidental de la Sierra de Guadalupe (Figura 1b). Mediante un análisis morfoestructural detallado, GarcíaPalomo et al. (2008), mostraron la importancia de las fallas en el relieve de la Sierra de las Cruces y definieron algunas fallas como: falla Otomí (E-W), fallas Río Hondo (NE-SW), fosa de Barrientos (E-W) y fallas Catedral (N-S) (ver Figura 1c). Adicionalmente, también se han reportado fallas con orientación E-W, como falla Xochimilco y falla La Pera, al norte y al sur de la Sierra Chichinautzin, respectivamente (Figura 1c); éstas presentan un desplazamiento importante, generando lineamientos observables en imágenes satelitales (Delgado-Granados et al., 1995; Alaniz-Álvarez y Nieto-Samaniego, 2005; Norini et al., 2006; García-Palomo et al., 2008; Arce et al., 2013; Lermo et al., 2016). Dentro de la cuenca de México se han definido dos sistemas de fallas principales: uno, ENE-WSW, representado por lineamientos paralelos a la Sierra de Santa Catarina (Figura 1c) y observados a diferentes profundidades del contacto entre rocas calcáreas del Cretácico y las rocas volcánicas del Cenozoico (Pérez-Cruz, 1988). A este mismo sistema pertenece la fosa de Santa Catarina, formada por dos fallas normales con una orientación ENE-WSW (Figura 1c), la cual fue definida por Arce et al. (2013) con la ayuda de las columnas litológicas descritas en los pozos profundos perforados al norte (Mixhuca y San Lorenzo Tezonco) y al sur (Tulyehualco) de la Sierra de Santa Catarina. El otro sistema de fallas que se ha reportado dentro de la cuenca, tiene una orientación NNW-SSE y también se ha definido con base en la variación de la profundidad del contacto entre rocas calcáreas y volcánicas. Gracias a dicha variación se pudo definir a la falla Mixhuca (Figura 1c), la cual es de tipo normal y parece acomodar un desplazamiento importante generando la fosa Roma, al oeste de la falla (Pérez-Cruz, 1988). La actividad de uno de estos dos sistemas de fallas dentro de la cuenca de México, es confirmada con los estudios sísmicos realizados en el centro de México, ya que se han reportado sismos relacionados a fallas normales que tienen una orientación ENE-WSW (Lermo et al., 2016). Estos eventos sugieren que este sistema puede ser activo, lo cual es congruente con lo observado en otras partes de la FVTM (Suter et al., 2001; Sunye-Puchol et al., 2015) y con la deformación actual del arco volcánico (Alaniz-Álvarez et al., 1998; Ferrari et al., 2011; Manea et al., 2013).

\section{METODOLOGÍA}

El estudio de la geometría de las fracturas en núcleos de perforación se realiza generalmente a través del análisis en una dimensión (1D), debido principalmente a la limitación relacionada con el diámetro del cilindro de la muestra de roca obtenida. Para el análisis son necesarios datos de apertura y espaciamiento entre fracturas, los cuales son obtenidos a través de una línea de muestreo (Figura 4). En ciertos casos se pueden hacer mapas de fracturas (análisis en 2D) en escalas del orden de 0.01-0.1 m²; sin embargo, esto dependerá de la distribución y número de fracturas (Vásquez-Serrano, 2013). Para este trabajo sólo se realizó el análisis en 1D a lo largo de un núcleo continuo de $9 \mathrm{~m}$ de longitud recuperado del pozo Agrícola Oriental 2C. El estudio tomó en cuenta una serie de parámetros para describir la distribución de las fracturas. La metodología para calcular el valor de estos parámetros y sus bases teóricas se describen a continuación.

\section{Análisis fractal de fracturas en 1D}

Los fractales son cuerpos geométricos que no tienen dimensiones enteras porque ocupan una fracción del espacio euclidiano (Mandelbrot, 1967). Se pueden describir a partir de un parámetro

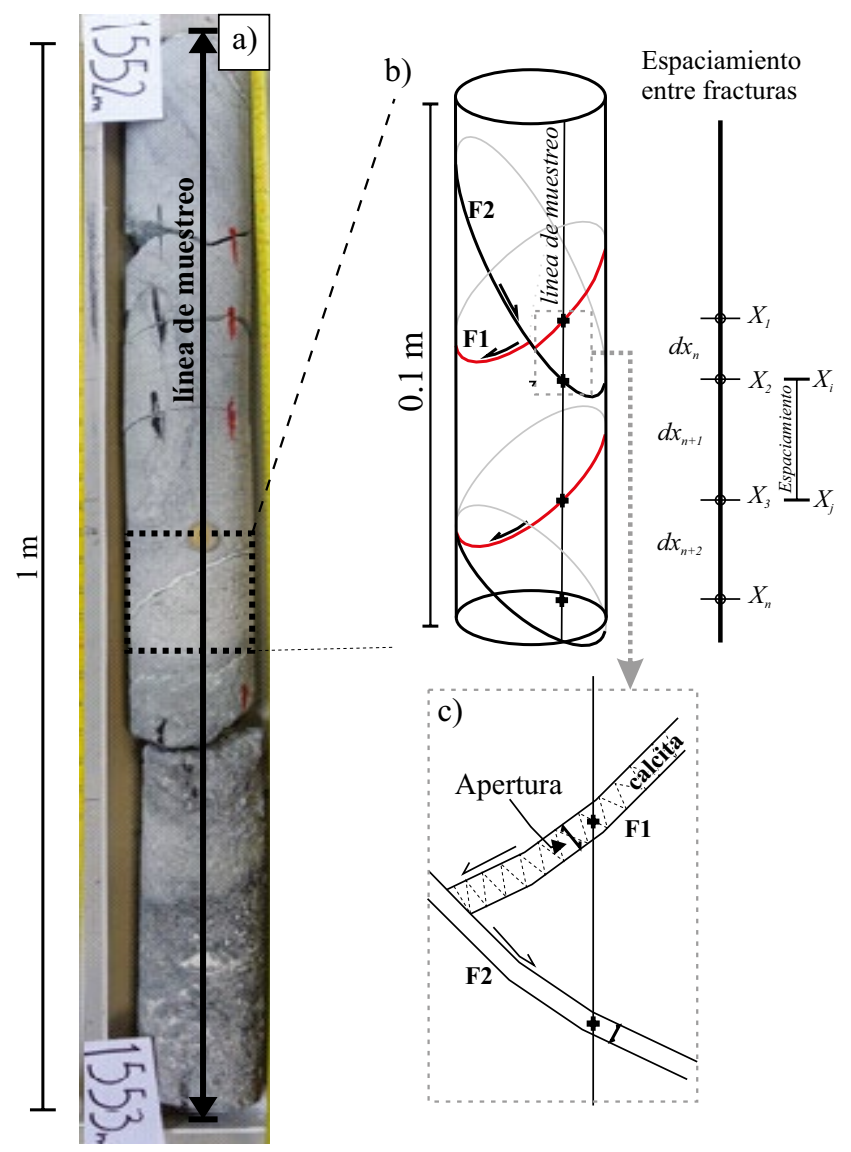

Figura 4. Definición de la apertura y el espaciamiento entre fracturas para los núcleos estudiados en el pozo Agrícola Oriental 2C. a) Ejemplo de la adquisición de datos a partir de una línea de muestreo de $1 \mathrm{~m}$ de longitud en la porción 1552-1553 m del núcleo estudiado. b) Adquisición de la distancia entre fracturas (espaciamiento) sin discriminar entre familias de fracturas (F1 y F2). c) Definición de la apertura y la manera de medirla (distancia entre las paredes de la fractura medida perpendicularmente a ellas). $X_{1}, X_{2}, X_{3} \ldots X_{n}$ son las coordenadas de los puntos de intersección entre las fracturas y la línea de muestreo, mientras que $d x_{n}, d x_{n+1}, d x_{n+2} \ldots$ son espaciamientos entre fracturas. 
llamado dimensión fractal (D), el cual va a tener un valor de entre 0 y 1 para una dimensión, de 1 a 2 para dos dimensiones y entre 2 y 3 en el caso tridimensional (Turcotte, 1992; Korvin, 1992; Barton y LaPointe, 1995). Una característica fundamental de los fractales es su invariancia en la escala, lo cual significa que su geometría se repite en un amplio rango de escalas (Mandelbrot, 1967; Barton y LaPonte, 1995). La dimensión fractal, al igual que otros parámetros como la dimensión de correlación, exponente de frecuencia acumulada, exponente de Lyapunov y coeficiente de variación pueden ayudar a caracterizar de manera apropiada a las fracturas observadas en 1D. Sumado a lo anterior también se puede conocer la intensidad de fracturas.

Dimensión fractal de caja (Dc). Una manera de conocer cómo ocupa el espacio un objeto fractal es mediante su dimensión fractal. Este parámetro se puede obtener a través de una metodología llamada conteo de cajas, la cual usa los datos de espaciamiento entre fracturas. Para obtener el valor de la dimensión se usa una línea fraccionada en distintos segmentos de tamaño $r$. Para cada $r$ se cuenta el número de segmentos que contiene por lo menos una fractura. Si se realiza un gráfico log-log del tamaño del segmento $r$ contra el número de segmentos que cortan a alguna fractura $(\mathrm{N})$, se observará la siguiente relación:

$$
N=r^{D c}
$$

donde $D c$ es la dimensión fractal de caja (Barton y LaPointe, 1995; Vásquez-Serrano, 2013).

\section{Dimensión de correlación $(v)$}

Es un parámetro que usa los datos de distancia entre fracturas (espaciamientos) y provee un cálculo matemático riguroso de la dimensión fractal (Davy et al., 1990). La dimensión de correlación se basa en la expresión propuesta por Grassberger y Procaccia (1983), en donde se tiene que:

$$
C(\gamma)=\lim _{N \rightarrow \infty}\left[\frac{1}{N^{2}} \sum_{i=1}^{N} \sum_{j=1}^{N} \theta\left(\gamma-\left|X_{i}-X_{j}\right|\right]\right.
$$

Aquí $\gamma$ es cualquier distancia propuesta, $\mathrm{X}=\left(\mathrm{X}_{1}, \mathrm{X}_{2}, \mathrm{X}_{3}, \ldots, \mathrm{X}_{\mathrm{n}}\right)$ es el conjunto de puntos (coordenadas) de fracturas cortadas por una línea de muestreo (Figura 4b), $\theta$ es la función de Heaviside, $\left|X_{i}-X_{j}\right|$ es el valor absoluto de la distancia entre pares de fracturas (espaciamiento) y $\mathrm{N}$ es el número de todos los posibles pares. En general, la dimensión de correlación cuenta el número total de posibles pares que tienen una distancia menor que $\gamma$. Tomando como base la expresión anterior, Grassberger y Procaccia (1983) demostraron que:

$$
C(\gamma) \sim \gamma^{v}
$$

Donde $v$ es la dimensión de correlación. Si $v \approx 0$ existirá sólo una ligera variación en los espaciamientos, es decir, estos se acercarán a ser periódicos (Riley et al., 2011).

\section{Exponente de frecuencia acumulada (Efa)}

Una manera de analizar los datos de espaciamiento y apertura es a través de la frecuencia acumulada. Ésta se basa en el concepto de fragmentación (frecuencia-tamaño), el cual ha sido explorado en diversos trabajos (Korvin, 1992; Gillespie et al., 1993; Cladouhos y Marrett, 1996; Marrett et al., 1999; Hooker et al., 2013). Los gráficos de espaciamiento o apertura contra logaritmo de frecuencia acumulada suelen seguir una ley de potencia.

$$
S=v^{E f a}
$$

En la ecuación anterior, $S$ es la frecuencia acumulada, Efa es el exponente de frecuencia acumulada y $v$ es la variable de interés (espaciamiento o apertura). Este parámetro da una medida de la distribución del tamaño, es decir, la frecuencia con la que se repite un tamaño determinado del espaciamiento o apertura.
Exponente de Lyapunov (Lv)

Este parámetro parte de la solución general de un sistema no lineal como el siguiente:

$$
x(t)=c_{1} e^{\lambda_{1} t} v_{1}+c_{2} e^{\lambda_{2} t} v_{2}
$$

Donde $c_{1}$ y $c_{2}$ son constantes, $\lambda_{1}$ y $\lambda_{2}$ son dos valores propios, mientras que $v_{1}$ y $v_{2}$ son los correspondientes vectores propios. Para un sistema dinámico no lineal, los valores propios muestran cómo dos puntos convergen (si son positivos) o divergen (si son negativos) exponencialmente. Entre los valores propios, existen algunos llamados exponentes de Lyapunov, de los cuales hay uno que caracteriza al sistema, conocido como exponente máximo de Lyapunov, $L v$ (Rosenstein $e t$ al., 1993). Existen tres casos para $L v$ : si es negativo, el sistema converge a un punto; si es cero, indica que el sistema es periódico y si es positivo el sistema será caótico. La determinación de $L v$ requiere conocer las ecuaciones diferenciales que gobiernan al sistema, lo cual es complicado de determinar en muchos sistemas naturales. Rosenstein et al. (1993) proponen una expresión para determinar $L v$ a partir de los datos de espaciamiento entre fracturas que no necesita de un conocimiento tan profundo para ser implementada.

$$
L v=\lim _{N \rightarrow \infty}\left(\frac{1}{N} \sum_{n=1}^{N} \log 2\left|\frac{d x_{n+1}}{d x_{n}}\right|\right)
$$

Aquí $N$ es el número de puntos de fracturas cortados en una línea de muestreo (Figura $4 \mathrm{~b}$ ), $d x_{n}$ es el espaciamiento entre fracturas y $d x_{n+1}$ es el espaciamiento próximo a $d x_{n}$. Cuando hay grandes fluctuaciones en el espaciamiento (grupos de fracturas), se tendrá una $L v>0$; por otro lado, si se tienen espaciamientos regulares, $L v$ se aproximará a cero.

\section{Coeficiente de variación ( $\mathrm{Cv}$ )}

Una medida de la distribución de las fracturas está dada por el coeficiente de variación, el cual es definido como el cociente de la desviación estándar entre el promedio de los espaciamientos (Cox y Lewis, 1966). Cuando los espaciamientos son regulares se tendrá una desviación estándar pequeña y por lo tanto un $C v \sim 0$. Por otro lado, cuando las fracturas están agrupadas y existen grandes espacios, la desviación estándar será grande y $C v>1$.

\section{Intensidad de fracturas}

Este parámetro mide la cantidad de fracturas por unidad de longitud (Mauldon et al., 2001; Nieto-Samaniego et al., 2003, 2005; Moreno-Sánchez y García-Cabrejo, 2006). Para medir la intensidad de fracturas se suelen usar líneas de muestreo rectas o circulares, donde se cuenta el número de fracturas que son cortadas por dicha línea (Moreno-Sánchez y García-Cabrejo, 2006). El valor de este parámetro se obtendrá dividiendo el número de fracturas cortadas entre la longitud de la línea de muestreo.

\section{Recolección de datos. Zona de falla en el núcleo recuperado del pozo Agrícola Oriental 2C}

El análisis de las fracturas se realizó en un núcleo continuo de 9 $\mathrm{m}$ de longitud, del pozo Agrícola Oriental 2C (Figura 1c, 19²3'43”N, $99^{\circ} 05^{\prime} 11^{\prime \prime} \mathrm{W}$ ), obtenido a una profundidad entre 1550 y $1559 \mathrm{~m}$ (Figura 3). Este núcleo está constituido básicamente por una roca volcánica de color gris claro, con algunas zonas de color verdoso, compacta, de textura porfídica, con algunos cristales reconocibles de plagioclasa, piroxeno, así como pirita diseminada y, en menor proporción, epidota (Figuras $3 \mathrm{~b}$ y $4 \mathrm{a}$ ). A pesar del grado de alteración, la mineralogía observada sugiere que se trata de una roca de composición andesítica. De manera particular, el núcleo presenta una gran cantidad de fracturas, algunas rellenas de calcita y otras abiertas (Figuras $3 \mathrm{~b}$ y $4 \mathrm{a}$ ).

Los datos se obtuvieron a través de una línea de muestreo de la misma longitud que el núcleo recuperado. El espaciamiento entre 
fracturas y la apertura fueron tomados sin diferenciar entre familias de fracturas con el propósito de observar el efecto que tiene la superposición de fracturas en su distribución y arreglo (Figuras $4 \mathrm{~b} y$ 4c). La línea de muestreo principal fue dividida en nueve partes para determinar las variaciones de los parámetros estudiados a lo largo del núcleo. También se tomaron datos de orientación de las fracturas (dirección de inclinación e inclinación), tomando como referencia un norte arbitrario, con el propósito de encontrar patrones de orientación en las fracturas y compararlos con la orientación de las principales fallas en la cuenca de México.

El espaciamiento y la apertura fueron medidos con una cinta métrica, con graduación en milímetros. En el caso de la apertura se realizó una corrección con respecto a la orientación e inclinación de las fracturas para obtener la distancia perpendicular a las paredes de la fractura (Figura 4c). La medición del espaciamiento no tomó en consideración la orientación e inclinación de las fracturas, debido principalmente a que se quiere representar la distribución de todas las fracturas cortadas en la porción del pozo estudiado, como si fuera una línea de muestreo.

Una vez obtenidos los datos, se aplicaron los métodos del análisis en 1D. En el caso del espaciamiento se calculó el exponente de la frecuencia acumulada, coeficiente de variación, dimensión de caja y correlación, exponente de Lyapunov, así como la intensidad de fracturas. Para la apertura sólo se estimó el exponente de la frecuencia acumulada. La obtención de estos parámetros se realizó a través de hojas de cálculo y mediante el uso del programa Frac1Dv1.1 escrito en Matlab por Vásquez-Serrano (2013).

\section{RESULTADOS}

\section{Descripción de fracturas en el núcleo recuperado}

Las fracturas encontradas en el núcleo son extensionales (Modo I) y de cizalla (Modo II) (Irwin, 1960). Estas últimas son más comunes en la porción más profunda (1557-1559 m), cerca del contacto con las rocas calcáreas, encontradas a partir de $1570 \mathrm{~m}$ de profundidad en el pozo Mixhuca (Pérez-Cruz, 1988). De acuerdo con la cantidad de fracturas, alteración hidrotermal en la roca, presencia de zonas de cizalla con una foliación incipiente y pequeñas fallas secundarias (Figura 3b), se sugiere la presencia de una zona de falla (dominada por fracturas) que afecta a las rocas del núcleo; estas evidencias coinciden con lo reportado por Caine et al. (1996) para zonas de falla. Sin embargo, es importante mencionar que fue posible distinguir dos familias de fracturas con base en sus relaciones de corte y características del relleno. Las fracturas F1 generalmente se encuentran rellenas de calcita y son paralelas a zonas con una foliación incipiente consistente en reducción del grano y recristalización de minerales. Mientras que las fracturas F2 están relacionadas a la falla principal y cortan a las fracturas F1. En su mayoría se encuentran abiertas o rellenas con material arcilloso y óxidos. Cerca del contacto entre rocas sedimentarias y volcánicas, estas fracturas $\mathrm{F} 2$ presentan estrías y crecimiento mineral en dirección del movimiento. Según su cinemática, las fallas secundarias (F2) están relacionadas a una falla normal (Figura 5).

\section{Orientación de las fracturas}

La orientación de las fracturas fue medida tomando como referencia un norte arbitrario único a lo largo de todo el núcleo estudiado. El objetivo principal de esta actividad fue identificar familias de fracturas mediante la proyección esférica de los datos y su representación en un diagrama de polos de planos de fracturas (Figura 5). Adicionalmente se construyó un diagrama de rosa donde se identificaron las principales tendencias en el rumbo de las fracturas (Figura 5). En el diagrama de polos de fracturas se puede observar la formación de dos grupos de fracturas (F1 y F2), lo cual fue confirmado con el diagrama de rosa (Figura 5). Tomando como referencia el norte arbitrario, la familia F1 tiene fracturas inclinadas principalmente hacia el NW, con un rumbo general ENE-WSW. Por otro lado, la familia F2, relacionada con la falla principal observada, tiene fracturas sub-verticales inclinadas hacia el SW con un rumbo promedio NW-SE. Estas dos familias también pueden ser identificadas en el núcleo recuperado, donde las fracturas de F1 (rellenas de calcita) cortan a las fracturas abiertas F2 (Figura 3b).

\section{Ajuste de funciones en los datos de espaciamiento y apertura}

Una de las primeras aproximaciones para analizar los datos es mediante su ajuste a alguna función en un gráfico que incluya la relación entre la variable (espaciamiento o apertura) y su frecuencia acumulada. En muchos trabajos se ha reportado que los datos de espaciamiento y apertura se adecuan bien a funciones como ley de potencia, exponencial o log-normal (Turcotte, 1992; Gillespie et al., 1993; Cladouhos y Marrett, 1996; Marrett et al., 1999; Vásquez-Serrano, 2013; Hooker et al., 2018), dependiendo en gran parte del sesgo en las mediciones. Sin embargo, en la mayoría de los estudios se ha sugerido que la función de ley de potencia es la que mejor se ajusta al espaciamiento y
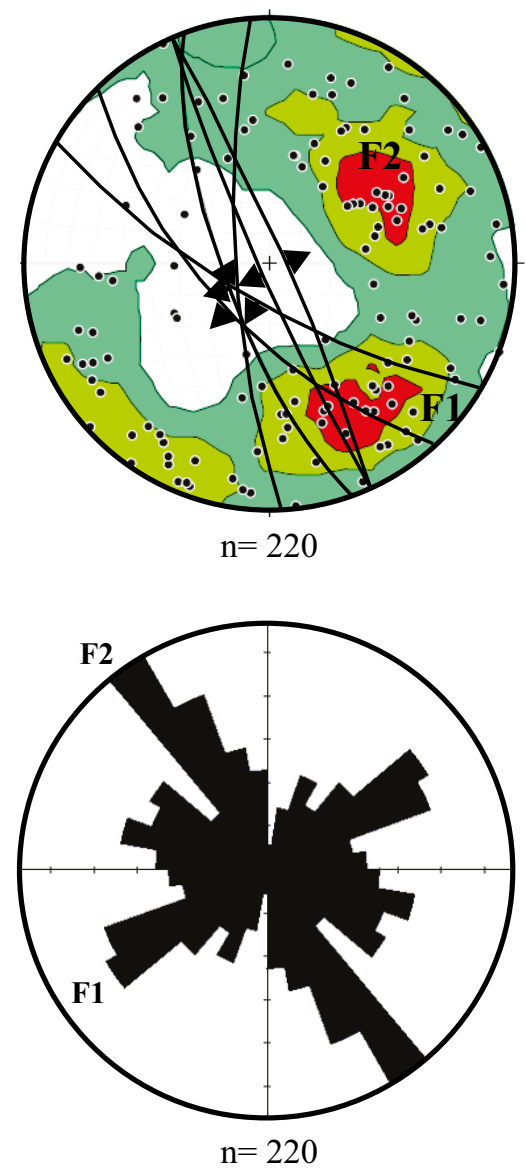

Figura 5. Proyección esférica (polos de planos de fracturas) y diagrama de rosa de las fracturas medidas en el núcleo recuperado del pozo Agrícola Oriental 2C. Los datos fueron obtenidos tomando un norte arbitrario debido a que el núcleo no se encuentra orientado. En el diagrama de polos, se incluyen trazas ciclográficas de planos de fallas secundarias medidas entre 1557 y 1559 m de profundidad relacionadas con la falla Agrícola Oriental. Nótese la presencia de dos familias de fracturas con diferente orientación ( F1 y F2), donde las fracturas F2 son paralelas a las fallas secundarias medidas. 
apertura, lo cual se refleja en su comportamiento fractal (Mandelbrot, 1967; Turcotte, 1992; Korvin, 1992; Barton y LaPointe, 1995). Con base en nuestras observaciones, las funciones que mejor describen la distribución del espaciamiento son ley de potencia con un exponente de frecuencia acumulada de -2.311 , y exponencial con un exponente de - 0.212. Ambas funciones tienen un coeficiente de variación $\mathrm{R}^{2}$ mayor a 0.95 (Figura 6a). Es importante resaltar que en un gráfico bilogarítmico del espaciamiento contra la frecuencia acumulada del espaciamiento, es posible observar la generación de dos pendientes en los datos: ambas se ajustan a una función de ley de potencia y tienen exponentes de frecuencia acumulada de -3.87 y -1.86 con un $\mathrm{R}^{2}$ mayor a 0.99 (Figura 6a).

Para el caso de la distribución de la apertura, las funciones que mejor se ajustan a los datos son exponencial, logarítmica y ley de potencia, con un $\mathrm{R}^{2}$ mayor a 0.88 (Figura $6 \mathrm{~b}$ ). Por otro lado, al hacer un gráfico bilogarítmico de la apertura contra su frecuencia acumulada, los datos trazan claramente dos pendientes como en el caso del espaciamiento. Ambas pendientes se ajustan muy bien a una función de ley de potencia con exponentes de frecuencia acumulada de -4.21 y -1.53 con un $\mathrm{R}^{2}$ de 0.9956 y 0.9704 respectivamente (Figura 6b).

\section{Variación de los parámetros medidos con la profundidad}

Para conocer la variación de los parámetros que caracterizan a las fracturas con la distancia (profundidad) a la falla, se dividió la línea de muestreo principal en nueve partes, cada una de un metro de longitud (Figuras 7 y 8). Con los datos de cada sub-línea de muestreo se calcularon los parámetros deseados siguiendo la metodología antes expuesta. Los resultados de dichos cálculos se presentan a continuación.

\section{Exponente de frecuencia acumulada}

Los datos de espaciamiento y apertura para cada sub-línea de muestreo se ajustan de manera adecuada a una ley de potencia (Ecuación 4), donde el exponente de frecuencia acumulada $(E f a)$ nos da una idea de la proporción del tamaño, es decir, de la cantidad de espaciamientos o aperturas con un rango de valores específico. Para la apertura se obtuvieron valores de Efa que van de 1.320 a 2.233 . La variación de este parámetro a lo largo del núcleo sugiere que cerca de la falla se tiene un valor alto, que disminuye gradualmente hasta llegar a una profundidad de $1555 \mathrm{~m}$, para después aumentar progresivamente hasta $1552 \mathrm{~m}$, donde vuelve a disminuir su valor (Tabla 1 y Figura 7). En el caso del espaciamiento entre fracturas, las nueve sub-líneas de muestreo tienen valores de Efa entre 1.270 y 2.333 (Tabla 1 y Figura $8 \mathrm{~b})$, mientras que su variación con la distancia a la falla muestra un aumento progresivo conforme se aleja de la falla hasta una distancia de $3 \mathrm{~m}$, donde disminuye $(1555 \mathrm{~m})$. A partir de este punto, el valor de Efa aumenta hasta 2.305 a una profundidad de $1552 \mathrm{~m}$ para después disminuir (Figura 8b).

\section{Intensidad de fracturas}

La intensidad de fracturas es un parámetro que proporciona una estimación de la cantidad de fracturas por unidad de longitud. En el caso de este estudio, los valores obtenidos de intensidad se encuentran en un rango comprendido entre 13 y 37 fracturas/m para las sub-líneas de muestreo analizadas (Tabla 1). La variación de este parámetro dentro del núcleo es muy importante, ya que idealmente tendría que disminuir progresivamente conforme nos alejamos de la falla (Riley et al., 2011; Brogi, 2011). Sin embargo, esto no es así en el núcleo estudiado, ya que existen fluctuaciones importantes a partir de $1556 \mathrm{~m}$ de profundidad que escapan del patrón ideal (Figura 8b).

\section{Dimensiones fractales}

La dimensión de caja $(D c)$ es un parámetro muy utilizado en el análisis fractal de mapas de fracturas. Sin embargo, se ha usado poco en variables unidimensionales como el espaciamiento (Barton y Larsen, 1985; Velde et al., 1990; Gillespie et al., 1993). Este parámetro tiene valores entre 0 y 1 , y mide la manera en que las fracturas ocupan la línea de muestreo, es decir, nos da una idea de la distribución y la densidad (Vásquez-Serrano, 2013). Los resultados del análisis realizado muestran
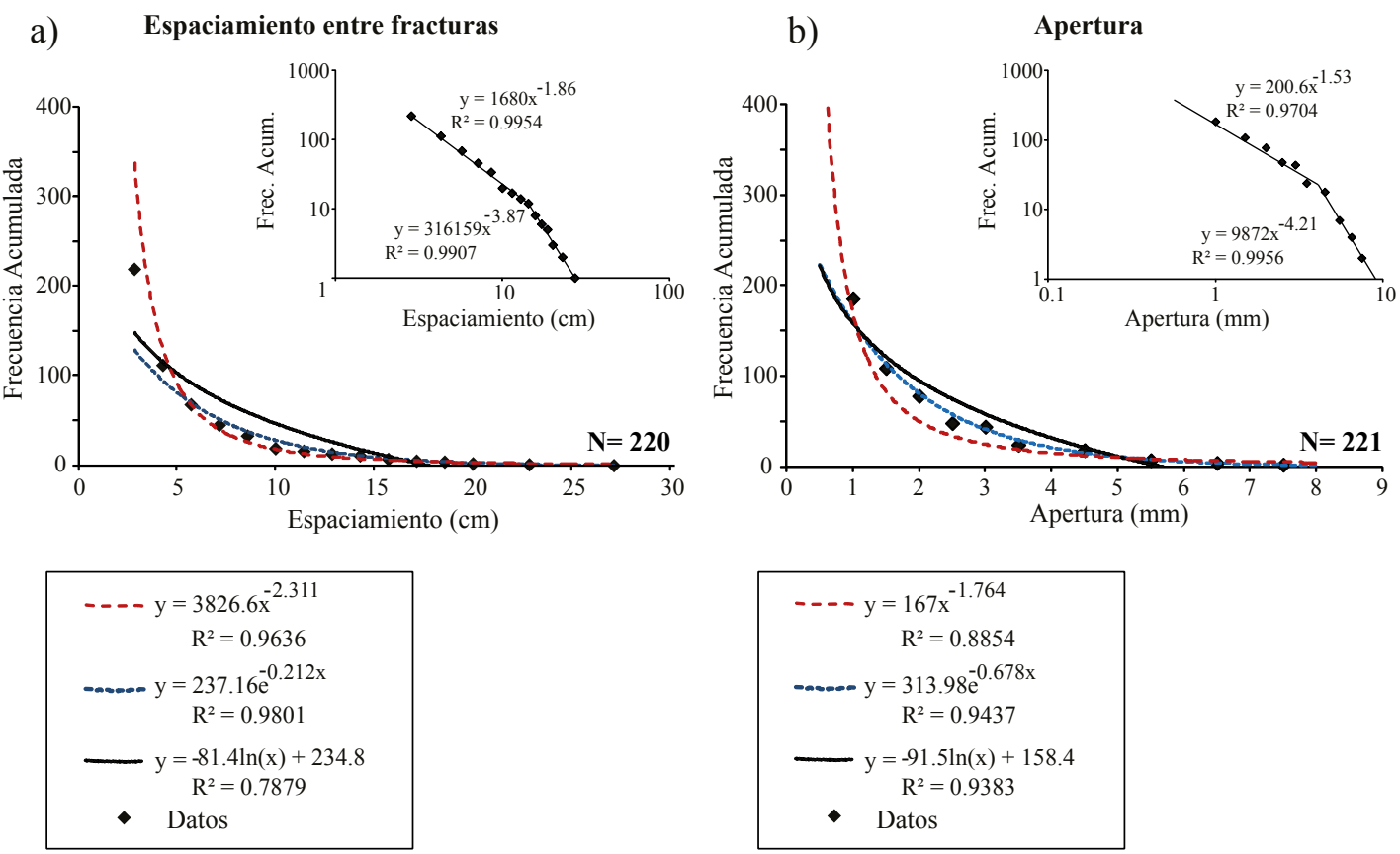

Figura 6. Gráficos que muestran la relación del espaciamiento (a) y la apertura (b) con su frecuencia acumulada. Se incluye también el ajuste de tres funciones (logarítmica, exponencial y ley de potencia) con sus coeficientes de correlación $\left(\mathrm{R}^{2}\right)$. En los gráficos bilogarítmicos se aprecian dos pendientes en los datos, que se ajustan muy bien a una función de ley de potencia. 


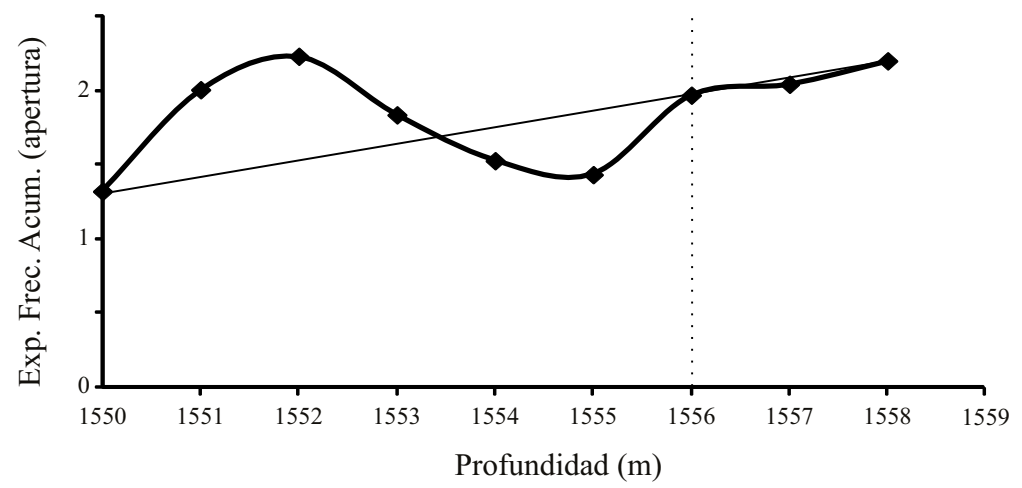

Figura 7. Variación del exponente de frecuencia acumulada de la apertura con la profundidad en el núcleo recuperado del pozo Agrícola Oriental 2C. Nótese la tendencia de los datos entre 1556 y $1559 \mathrm{~m}$, con picos por abajo y por arriba de esta tendencia en el segmento entre 1550 y $1556 \mathrm{~m}$.

que la $D c$ tiene valores entre 0.44 y 0.73 (Tabla 1 ). En lo que se refiere a la variación de la $D c$ dentro del núcleo, se observa una disminución progresiva con la distancia a la falla, hasta $1555 \mathrm{~m}$ de profundidad, donde aumenta hasta $1552 \mathrm{~m}$ para después disminuir nuevamente a $1550 \mathrm{~m}$ (Figura 8b).

Por otro lado, la dimensión de correlación (v) proporciona información acerca de la distribución de las fracturas, es decir, se mide la probabilidad de que un espacio entre fracturas tenga una distancia $\gamma$ (Ecuación 3) (Riley et al., 2011). Para las fracturas estudiadas en este trabajo, la dimensión de correlación tiene valores que están entre 0.27 y 0.58 , mientras que su variación con la profundidad sugiere una disminución conforme se incrementa la distancia a la falla hasta $1556 \mathrm{~m}$, donde aumenta un poco hasta $1554 \mathrm{~m}$ para posteriormente disminuir gradualmente por los siguientes $3 \mathrm{~m}$, donde finalmente aumenta en $1550 \mathrm{~m}$ (Figura 8b).

\section{Exponente de Lyapunov}

El exponente de Lyapunov (lv) se relaciona con el arreglo que tienen las fracturas en una línea de muestreo (Riley et al., 2011). De acuerdo con los resultados obtenidos, el valor de este parámetro se encuentra en un rango que va de 1.30 a 1.79 para las nueve sub-líneas de muestreo donde se realizó el análisis (Tabla 1). La variación que tiene este parámetro dentro del núcleo sugiere que el valor más grande del exponente de Lyapunov se encuentra cerca de la falla (Figura $8 b)$. El patrón observado en este parámetro es muy similar al que se puede ver en la dimensión de correlación, con una disminución de su valor a partir de la falla, hasta $1556 \mathrm{~m}$ de profundidad, para después mantenerse más o menos constante. Los resultados muestran que $l v>1$ en promedio para todos los casos, lo cual sugiere que la agrupación de estas estructuras parece ser el arreglo más común (Figura 8a).

\section{Coeficiente de variación}

El coeficiente de variación $(C v)$ es otro parámetro que permite caracterizar el arreglo que tienen las fracturas a través de su espaciamiento. Los resultados en este trabajo muestran que las fracturas cortadas en el pozo Agrícola Oriental 2C tienen un Cv entre 0.77 a 1.37 (Tabla 1). La variación de $C v$ con la profundidad permite observar que su valor aumenta de manera progresiva a partir de la falla hasta 1556 $\mathrm{m}$, donde disminuye progresivamente en los próximos dos metros para después tener un valor más o menos constante (Figura $8 \mathrm{~b}$ ).

\section{DISCUSIÓN}

\section{Variación del valor de los parámetros estudiados}

La deformación en una falla suele concentrarse en un plano y disminuir conforme aumenta la distancia al sitio de máxima deformación. Este comportamiento se ha observado en las rocas y experimentos (numéricos y analógicos), y ha sido sintetizado en modelos teóricos de deformación de fallas (Ramsay y Huber, 1987; Passchier y Trouw, 1996). En un caso normal, la intensidad de fracturas tendría que disminuir conforme aumenta la distancia a la falla (Figura 8b); sin embargo, esto puede ser alterado por la presencia de un grupo de fracturas preexistentes que afecten a las rocas dentro de la zona de cizalla nueva (Brogi, 2011). De acuerdo con los resultados obtenidos en este trabajo, es posible observar que el patrón de intensidad

Tabla1. Valores de los parámetros estimados en el análisis de fracturas del Pozo Agrícola Oriental 2C.

\begin{tabular}{|c|c|c|c|c|c|c|c|}
\hline \multirow[t]{2}{*}{ Profundidad } & \multicolumn{6}{|c|}{ Espaciamiento } & \multirow{2}{*}{$\begin{array}{c}\text { Apertura } \\
\text { Exponente } \\
\text { Frec. Acum. }\end{array}$} \\
\hline & $\begin{array}{l}\text { Exponente } \\
\text { Frec. Acum. }\end{array}$ & $\begin{array}{l}\text { Dimensión } \\
\text { de caja }\end{array}$ & $\begin{array}{c}\text { Dimensión } \\
\text { de correlación }\end{array}$ & $\begin{array}{l}\text { Exponente } \\
\text { de Lyapunov }\end{array}$ & $\begin{array}{l}\text { Coeficiente } \\
\text { de variación }\end{array}$ & $\begin{array}{l}\text { Intensidad } \\
\text { de fracturas }\end{array}$ & \\
\hline 1550 & 1.739 & 0.44 & 0.48 & 1.49 & 0.77 & 13 & 1.32 \\
\hline 1551 & 2.065 & 0.65 & 0.35 & 1.29 & 1.09 & 22 & 2.007 \\
\hline 1552 & 2.333 & 0.73 & 0.43 & 1.18 & 0.81 & 31 & 2.233 \\
\hline 1553 & 2.175 & 0.72 & 0.43 & 1.18 & 0.92 & 31 & 1.837 \\
\hline 1554 & 1.534 & 0.66 & 0.53 & 1.3 & 0.8 & 36 & 1.527 \\
\hline 1555 & 1.27 & 0.62 & 0.47 & 1.54 & 1.01 & 37 & 1.433 \\
\hline 1556 & 2.305 & 0.68 & 0.27 & 1.3 & 1.37 & 25 & 1.97 \\
\hline 1557 & 2.224 & 0.7 & 0.29 & 1.25 & 1.05 & 27 & 2.044 \\
\hline 1558 & 1.711 & 0.72 & 0.58 & 1.79 & 0.81 & 32 & 2.202 \\
\hline
\end{tabular}


de fracturas observado en el núcleo recuperado del pozo Agrícola Oriental 2C, escapa de la tendencia común en una zona de cizalla frágil (Figura 8b). La disminución progresiva de la cantidad de fracturas con la distancia a la falla se observa sólo en los primeros 3 metros (Figura $8 \mathrm{~b}$ ), probablemente debido a que a esa distancia se encuentra la máxima deformación relacionada con la falla. A partir de $1556 \mathrm{~m}$ de profundidad, la variación anómala de intensidad de fracturas se debe a la presencia de dos grupos de fracturas superpuestas (Figura 8b), lo cual altera de manera notable la cantidad de fracturas por unidad de longitud (Brogi, 2011). El incremento en la cantidad de fracturas no depende del relleno de la familia F1, ya que las familias de fracturas encontradas (F1 y F2) tienen orientaciones muy distintas (Figura 5). Esta característica descarta la posibilidad de que las fracturas F2 sean producto de la reactivación de una falla relacionada con las fracturas F1, lo cual es también soportado con las relaciones de corte y la orientación de las fracturas (Figuras 3 y 5 ).

Un aspecto interesante tratado en este trabajo y que no se ha explorado del todo en la literatura (Laubach et al., 2018), es conocer la relación que guarda la intensidad de fracturas con parámetros como la dimensión fractal (caja y correlación), exponente de Lyapunov, coeficiente de variación y exponente de frecuencia acumulada del espaciamiento y apertura. Adicionalmente, es de gran importancia entender cuál es la variación de dichos parámetros con la distancia a una falla y la influencia de la superposición de dos familias de fracturas (F1 y F2). Con base en los resultados, es posible establecer que la dimensión de caja se aproxima al patrón observado en la intensidad de fracturas (Figura 8a), lo cual puede ser interpretado como un efecto de la variación en la cantidad de fracturas. Este comportamiento ha sido propuesto en otros trabajos para la dimensión de caja en 2D, donde su valor se correlaciona muy bien con la densidad de fracturas (número de fracturas por unidad de área) (Barton y LaPointe, 1995; Vásquez-Serrano, 2013).

En lo que se refiere a la variación de los otros parámetros a lo largo del núcleo, éstos muestran un patrón particular en los primeros 3 metros de distancia a la falla, probablemente reflejando la zona de máxima influencia de dicha falla (Figura 8b). Dentro de esta distancia, el exponente de frecuencia acumulada del espaciamiento y el coeficiente de variación aumentan a partir de la falla, mientras que la dimensión de correlación y exponente de Lyapunov disminuyen (Figuras 8b y 9b). El valor de estos dos últimos parámetros es controlado principalmente por la distribución y el arreglo que tienen las fracturas, con lo cual los datos pueden interpretarse como una disminución en el grado de agrupamiento de las fracturas con la distancia (Figura 8b). Respecto al patrón que presenta el exponente de frecuencia acumulada del espaciamiento y el coeficiente de variación, es muy probable que se deba al aumento en el número de espaciamientos grandes $(\sim 20-27 \mathrm{~cm})$ con la distancia a la falla, lo cual puede ser normal, ya que al alejarse de la zona de máxima deformación las fracturas son escasas y están separadas a mayor distancia (Riley et al., 2011).

Entre $1550 \mathrm{~m}$ y $1556 \mathrm{~m}$ de profundidad, el valor de los parámetros estimados no sigue una tendencia definida, existen fluctuaciones que dependen de la cantidad de fracturas, el arreglo que tienen y la distribución que presentan. Existe una concentración importante de fracturas entre $1552 \mathrm{~m}$ y $1554 \mathrm{~m}$, reflejado en el marcado aumento en la intensidad de fracturas, dimensión de caja y exponente de frecuencia acumulada del espaciamiento. Así mismo, también se relaciona con una disminución sutil en la dimensión de correlación y exponente de Lyapunov. Esta concentración de fracturas no está relacionada con la falla que generó a las fracturas F2, ya que al parecer a esta distancia la influencia de la falla en la cantidad de fracturas no es tan marcada (Figura 8b). Por lo tanto, el aumento en la intensidad de fracturas entre $1552 \mathrm{~m}$ y $1554 \mathrm{~m}$ se explica por la suma de las dos familias de fractu- ras (F1 y F2) con una importante contribución de la familia F1. Este comportamiento demuestra el papel que tienen las fracturas preexistentes (F1) en el aumento en la cantidad de fracturas y el cambio en la distribución y el arreglo ante un nuevo evento de fracturamiento (F1).

En una zona de falla, la variación en la apertura de las fracturas con la distancia a la falla es un reflejo de la variación de la deformación acomodada a través de extensión, ya que en sitios cercanos a la falla habrá fracturas con aperturas más grandes en comparación con fracturas localizadas a distancias mayores (Ramsay, 1967; Hooker et al., 2013; Laubach et al., 2018). Al igual que los demás parámetros, el exponente de frecuencia acumulada de la apertura exhibe un patrón distintivo (Figura 7) entre $1556 \mathrm{~m}$ y $1559 \mathrm{~m}$, representado por una disminución progresiva al aumentar la distancia a la falla; esto indica que la cantidad de fracturas con aperturas grandes $(\sim$ 6-8 $\mathrm{mm}$ ) disminuye progresivamente con la distancia (Figura 7). A partir de $1556 \mathrm{~m}$ y hasta $1550 \mathrm{~m}$, el valor de este parámetro presenta fluctuaciones, teniendo un mínimo en 1555 m y un máximo en 1552 $\mathrm{m}$. El pico mínimo representaría un conjunto de fracturas dominado por aperturas pequeñas $(\sim 1-2 \mathrm{~mm})$, mientras que el pico máximo se relaciona con fracturas con aperturas más grandes $(\sim 4 \mathrm{~mm})$. El aumento en la apertura de las fracturas a $1552 \mathrm{~m}$ está relacionado con la familia de fracturas F1, ya que a esta distancia la influencia de la falla que genera las fracturas F1 no es tan marcada. Al parecer a 1552 m existe una concentración de la deformación por extensión, ligada con el evento que formó las fracturas F1, sin un desplazamiento importante por cizalla como se observa en las fracturas F2 cercanas a la falla (Figuras $3 \mathrm{~b}$ y $8 \mathrm{~b}$ ).

\section{Dos familias de fracturas superpuestas y su relación con las fallas de la cuenca de México}

Las rocas ubicadas dentro de la cuenca de México se encuentran afectadas principalmente por dos sistemas de fallas, NNW-SSE y ENEWSW (Lermo et al., 2016, Figura 1c; Alaniz-Álvarez et al., 1998). El sistema NNW-SSE es más antiguo, con un inicio en su actividad antes del Oligoceno que probablemente se extendió hasta el Mioceno (AlanizÁlvarez y Nieto-Samaniego, 2005; García-Palomo et al., 2006), mientras que el sistema ENE-WSW es de actividad cuaternaria principalmente (Alaniz-Álvarez y Nieto-Samaniego, 2005). También ha sido reportado un sistema NE-SW al norte de la cuenca (García-Palomo, et al., 2006); sin embargo, en el sitio donde se ubica el pozo Agrícola Oriental 2C no existe evidencia de la presencia de este sistema (Figura 1c).

Con base en la ubicación del pozo Agrícola Oriental 2C, es posible observar en el mapa de la Figura 1c, que dicho pozo se encuentra en un sitio cercano al cruce de dos fallas principales: la falla Mixhuca con un rumbo NW-SE (Pérez-Cruz, 1988) y la falla del flanco norte de la fosa de Santa Catarina con un rumbo ENE-WSW (Arce et al., 2013).

Tomando como referencia los datos de orientación de fracturas obtenidos en este trabajo, es posible distinguir dos familias de fracturas (F1 y F2). Sin embargo, no es posible determinar de manera simple cuáles de las fallas reportadas (NNW-SSE o ENE-WSW) se relacionan de manera específica con cada familia, debido a que el núcleo recuperado no está orientado. Una manera de resolver este problema sería hacer una aproximación midiendo los ángulos entre los rumbos de las fallas principales cercanas (Figuras 1c y 2) y comparándolos con los ángulos entre las familias de fracturas en un diagrama de rosa (Figura 6). La dificultad de hacer esta comparación radica en que el ángulo que hay entre el rumbo de las familias es de $\sim 90^{\circ}$ (Figura 6), mismo ángulo que existe entre los rumbos de las fallas Mixhuca y la del flanco norte de la fosa de Santa Catarina (Figura 1c). Sin embargo, si se toma en cuenta las relaciones de corte (F2 más joven que F1) y las características de las fracturas estudiadas (F1 con fracturas rellenas de calcita y F2 con fracturas abiertas que tienen evidencia de desplaza- 
a)

Distribución de fracturas

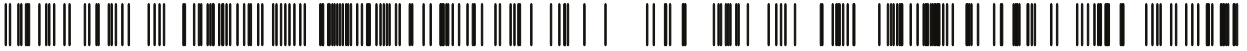

b)

No. de fracturas: 220

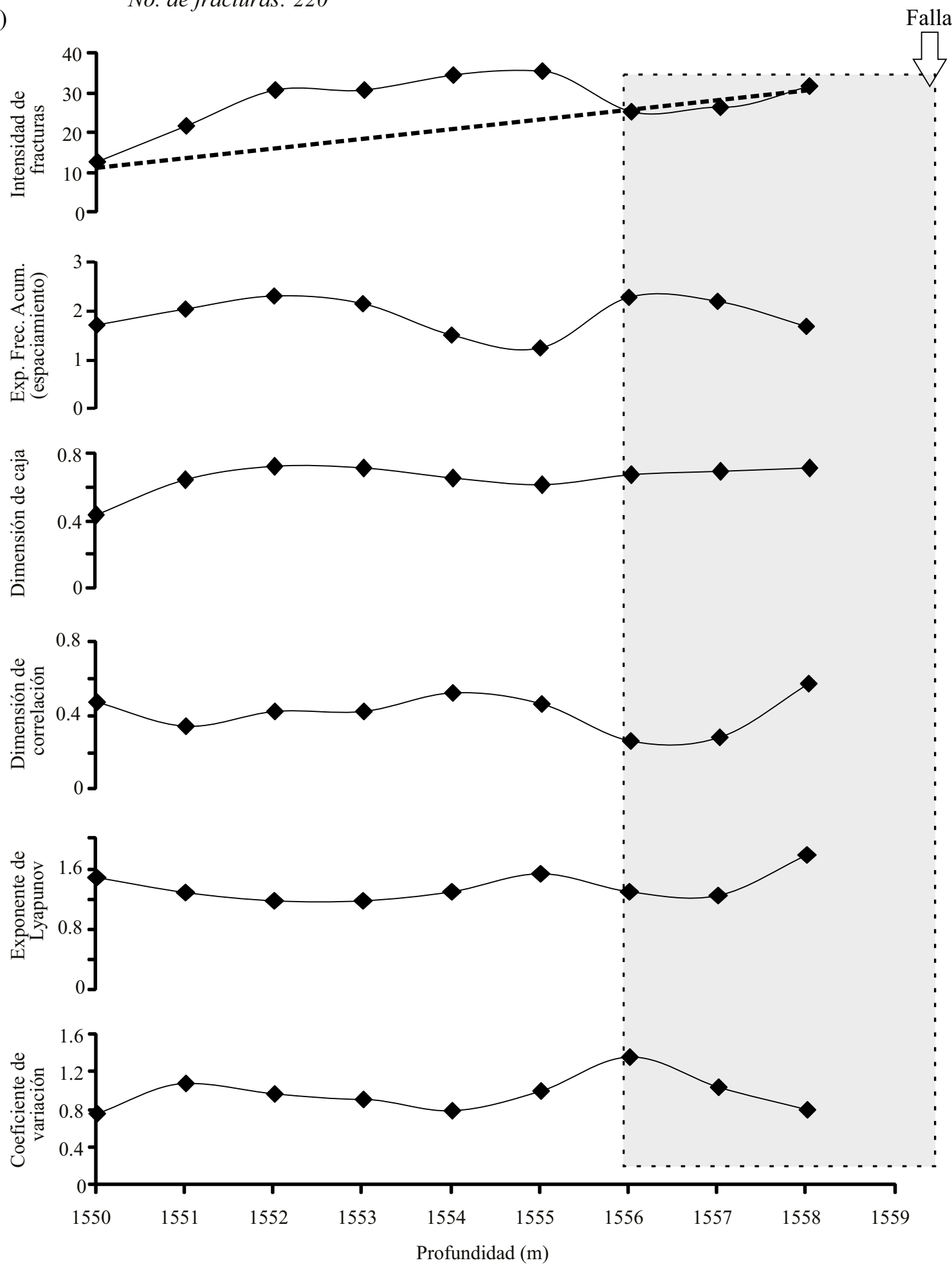

Figura 8. a) Distribución de fracturas (F1 + F2) a lo largo de $9 \mathrm{~m}$ de longitud del núcleo recuperado. b) Comparación de los parámetros estimados en este trabajo y su variación con la profundidad. El rectángulo gris, muestra el área de influencia que tiene la falla Agrícola Oriental en la distribución y cantidad de fracturas. Adicionalmente se incluye una línea de tendencia en la variación de la intensidad de fracturas formada entre 1556 y 1559 m de profundidad, donde se observa claramente que, en la zona entre 1550 y $1556 \mathrm{~m}$ de profundidad, los valores de intensidad escapan de dicha tendencia. 
miento y son paralelas a la falla cortada en el pozo), así como la edad y ubicación de los sistemas de fallas en la cuenca de México (sistema NNW-SSW más viejo que el sistema ENE-WSW), es posible tener una buena aproximación. A partir de estas consideraciones, lo más probable es que la falla cortada pertenezca al flanco norte de la fosa de Santa Catarina, por lo que se le puede asignar el nombre informal de falla Agrícola Oriental (Figuras 1c y 9a).
Las observaciones realizadas en este estudio permiten establecer que el contacto entre rocas volcánicas de probable edad del Oligoceno (andesitas) y las rocas sedimentarias del Cretácico (calizas), es a través de una falla normal con un rumbo ENE-WSW (Figura 9a). Esta observación es importante, ya que no se había reportado con certeza la naturaleza de este contacto. En el pozo Mixhuca, ubicado a unos metros del pozo Agrícola Oriental 2C (Figura 2), el contacto entre estas dos

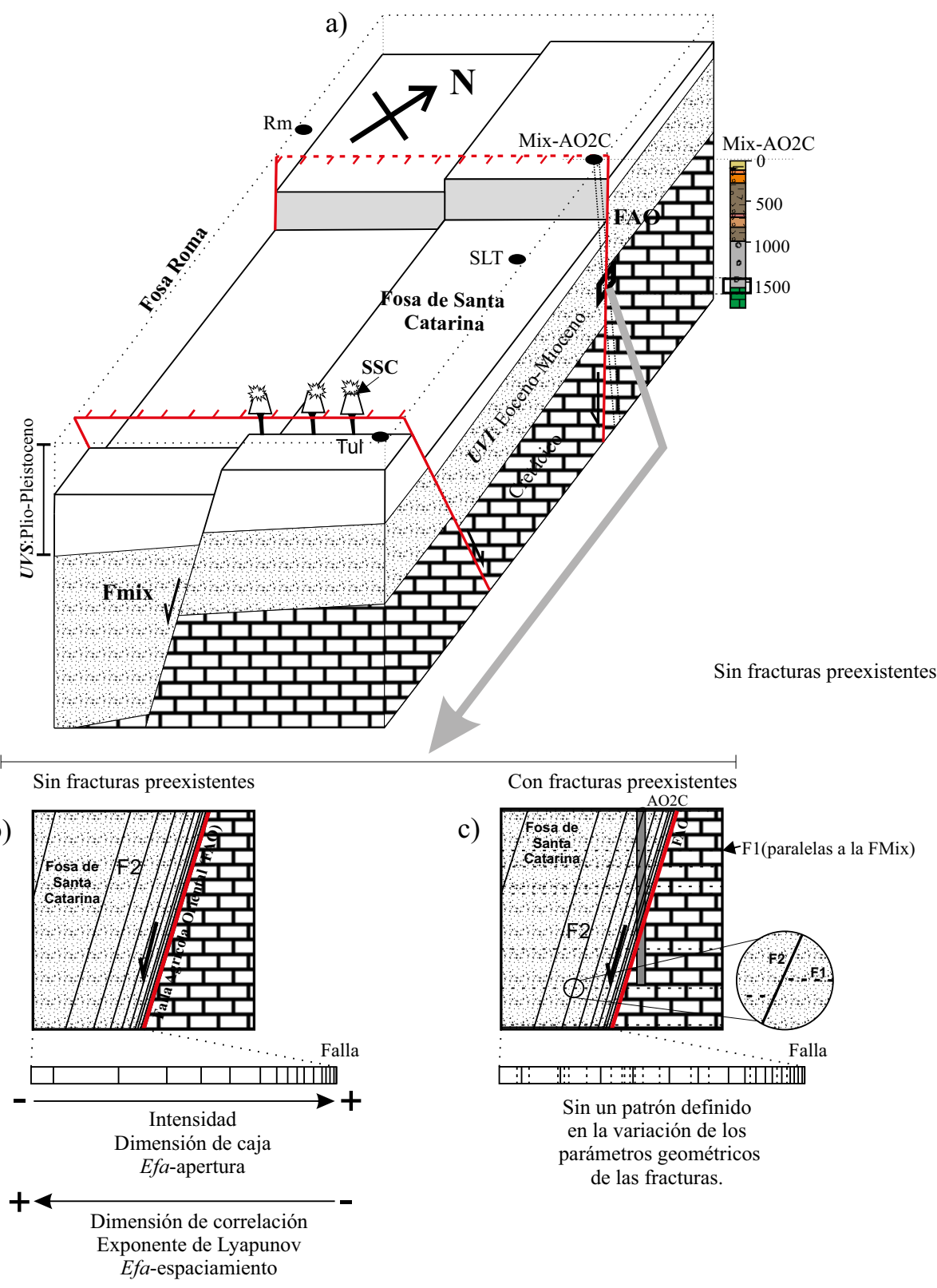

Figura 9. a) Modelo representativo de la situación estructural del centro de la cuenca de México (sin escala horizontal). En este trabajo, se interpreta que la falla Agrícola Oriental (FAO) pone en contacto a rocas volcánicas del Oligoceno (pertenecientes a la Unidad Volcánica Interior (UVI) del Eoceno-Mioceno según Pérez Cruz, 1988) con rocas calcáreas del Cretácico (Pérez-Cruz, 1988). Esta falla se ubica en el flanco norte de la fosa de Santa Catarina (Arce et al., 2013), la cual tiene un rumbo ENE-WSW, paralelo al rumbo de la Sierra de Santa Catarina (SSC). El sistema de fallas ENE-WSW corta a la falla Mixhuca (FMix), la cual forma parte de la fosa Roma con rumbo NNW-SSE y se localiza al oeste de los pozos Mixhuca y Agrícola Oriental 2C (Mix-AO2C). El modelo incluye también la ubicación de los pozos Tulyehualco (Tul), San Lorenzo Tezonco (SLT) y Roma (Rm), así como la columna estratigráfica descrita en el pozo Mixhuca por PEMEX (Pérez-Cruz, 1988); UVS es la Unidad Volcánica Superior (Pérez-Cruz, 1988). b) Familia de fracturas (F2) generada sólo por la FAO, en donde los patrones de intensidad de fracturas, dimensión de caja y $E f a$ de la apertura aumentan su valor al acercarse a la falla; mientras que la dimensión de correlación, exponente de Lyapunov y $E f a$ del espaciamiento disminuyen. c) Efecto de la superposición de dos familias de fracturas (F1 y F2) en la variación de la distribución, arreglo y cantidad de fracturas observadas en el núcleo recuperado del pozo Agrícola Oriental 2C. En este caso, los parámetros estimados no siguen un patrón definido, como se observa en una zona de falla común (Brogi, 2011). 
unidades había sido reportado como una discordancia (Pérez-Cruz, 1988), aunque no existen descripciones detalladas del estado de las rocas en dicho contacto que ayuden a apoyar esta idea.

Es evidente que en las rocas cortadas por el pozo Agrícola Oriental $2 \mathrm{C}$, cerca del contacto entre rocas sedimentarias e ígneas, se encuentran afectadas por dos familias de fracturas (F1 y F2) producidas por los dos sistemas de fallas reportados en el centro de la cuenca de México (Figuras 1c y 9a). La presencia de estos grupos de fracturas altera los patrones normales en la cantidad, distribución y arreglo de las fracturas dentro de una zona de cizalla, lo cual se refleja en la variación particular de los parámetros calculados en este trabajo (Brogi, 2011). Es posible observar, por ejemplo, la generación de dos pendientes en el gráfico bilogarítmico de la frecuencia acumulada del espaciamiento y la apertura (Figura 6), lo cual puede interpretarse como la presencia de dos familias de fracturas relacionadas a diferentes eventos de deformación (Barton y LaPointe, 1995). Esta interpretación es congruente con los datos de orientación y las relaciones de corte de las fracturas (Figuras 3 b y 5). Tomando en cuenta los eventos de deformación reportados para el centro de la cuenca de México, las fracturas F1 estarían relacionadas con el sistema de fallas NNW-SSE de edad Oligoceno-Mioceno, mientras que las fracturas F2 serían formadas a partir de la actividad cuaternaria del sistema de fallas ENE-WSW.

Por otro lado, la combinación de estos dos sistemas de fracturas es fundamental en el entendimiento del proceso de flujo de fluidos en fracturas localizadas en una zona de cizalla, ya que aumentan la permeabilidad secundaria a través de una conectividad más eficiente, producida por un incremento en la intensidad de fracturas y por la variación anómala en la distribución y arreglo que tienen estas estructuras (Figuras 8 b y 9c). En el futuro será importante tomar en cuenta los sitios en donde dos fallas se cortan, para tener mejores resultados en exploración de aguas subterráneas dentro de la cuenca de México.

\section{CONCLUSIONES}

El análisis de fracturas geológicas es fundamental para entender procesos complejos como el flujo de fluidos a través de estas estructuras, lo cual es vital para la exploración de petróleo, vetas minerales o agua subterránea. En este trabajo fue posible determinar en una zona de cizalla, la variación de la cantidad, distribución y arreglo de las fracturas encontradas en un núcleo recuperado entre 1550 y 1559 m de profundidad del pozo Agrícola Oriental 2C, localizado dentro de la Ciudad de México. De acuerdo con los resultados obtenidos, es posible establecer que, en una zona de cizalla, al acercarse a la falla principal, existe un aumento en la intensidad de fracturas, dimensión fractal de caja y correlación, exponente de Lyapunov y exponente de frecuencia acumulada de la apertura. Un comportamiento contrario se observa en parámetros como coeficiente de variación y exponente de frecuencia acumulada del espaciamiento. Estos patrones son alterados con la presencia de fracturas preexistentes que cambian la tendencia esperada, incrementando el número de fracturas y produciendo una nueva distribución.

La zona de cizalla encontrada en las rocas cortadas por el pozo Agrícola Oriental a $1559 \mathrm{~m}$ de profundidad, pertenece a una falla (llamada en este trabajo falla Agrícola Oriental) con un rumbo probable ENE-WSW que se localiza en el flanco norte de la fosa de Santa Catarina y que pone en contacto rocas calcáreas del Cretácico con rocas ígneas del Oligoceno. Estas características hacen de esta estructura una pieza importante para entender la geometría y distribución de las fracturas a profundidad y su relación con la tectónica de la cuenca de México.

\section{AGRADECIMIENTOS}

Agradecemos al Sistema de Aguas de la Ciudad de México por proporcionar el núcleo recuperado del Pozo Agrícola Oriental 2C para la realización de este estudio. Gracias a la Lic. Sandra González Luz por su apoyo en la elaboración del modelo digital de elevación utilizado para el mapa de localización. Este trabajo fue financiado por el proyecto PAPIIT IN102317 (a cargo del Dr. José Luis Arce Saldaña) y por los Convenios: No. 0296-1O-ED-DT-1-16-1928 y No. 0446-1O-EDDT-1-17-1928 entre el SACMEX, Gobierno de la Ciudad de México y el Instituto de Geología, UNAM. Agradecemos también la revisión y los comentarios al manuscrito del Dr. Gabriel Chávez Cabello, del Dr. Enrique Cabral Cano y de un revisor anónimo.

\section{REFERENCIAS}

Agustín-Flores, J., Siebe, C., Guilbaud, M.N., 2011, Geology and geochemistry of Pelagatos, Cerro del Agua, and Dos Cerros monogenetic volcanoes in the Sierra Chichinautzin Volcanic Field, south of México City: Journal of Volcanology and Geothermal Research, 201, 143-162.

Alaniz-Álvarez, S.A., Nieto-Samaniego, A.F., 2005, El sistema de fallas TaxcoSan Miguel de Allende y la Faja Volcánica Transmexicana, dos fronteras tectónicas del centro de México activas durante el Cenozoico: Boletín de la Sociedad Geológica Mexicana, Volumen Conmemorativo del Centenario. Grandes Fronteras Tectónicas de México, LVII(1), 65-82.

Alaniz-Álvarez, S.A., Nieto-Samaniego, A.F., Ferrari, L., 1998, Effect of the strain rate in the distribution of monogenetic and polygenetic volcanism in the Transmexican Volcanic Belt: Geology, 26, 591-594.

Alaniz-Álvarez, S.A., Nieto-Samaniego, A.F., Reyes-Zaragoza, M.A., OrozcoEsquivel, M.T., Ojeda-García, A.C., Vasallo-Morales, L.F., 2001, Estratigrafía y deformación de la región San Miguel de Allende-Querétaro: Revista Mexicana de Ciencias Geológicas, 18(1), 129-148.

Alaniz-Álvarez, S.A., Nieto-Samaniego, A.F., Orozco-Esquivel, M.T., VasalloMorales, L.F., Xu, S.S., 2002, El Sistema de Fallas Taxco- San Miguel de Allende: Implicaciones en la deformación post-Eocénica del centro de México: Boletín de la Sociedad Geológica Mexicana, 55, 12-29.

Arce, J.L., Macías, R., García-Palomo, A., Capra, L., Macías, J.L., Layer, P., Rueda, H., 2008, Late Pleistocene flank collapse of Zempoala Volcano (Central Mexico) and the role of fault reactivation: Journal of Volcanology and Geothermal Research, 177, 944-958.

Arce, J.L., Layer P.W., Morales-Casique, E., Benowitz, J.A., Rangel, E., Escolero, O.A., 2013, New constraints on the subsurface geology of the Mexico City Basin: The San Lorenzo Tezonco deep well, on the basis of ${ }^{40} \mathrm{Ar} /{ }^{39} \mathrm{Ar}$ geochronology and whole-rock chemistry: Journal of Volcanology and Geothermal Research, 266, 34-49.

Arce, J.L., Layer, P., Martínez, I., Salinas, J.I., Macías-Romo, M.C., MoralesCacique, E., Benowitz, J., Escolero, O.A., Lenhardt, N., 2015, Geología y estratigrafía del pozo profundo San Lorenzo Tezonco y sus alrededores, sur de la Cuenca de México: Boletín de la Sociedad Geológica Mexicana, 67(2), 123-143.

Babadagli, T., 2001, Fractal analysis of 2-D fracture networks of geothermal reservoirs in southwestern, Turkey: Journal of Volcanology and Geothermal Research, 112, 83-103.

Bahat, D., Rabinovitch, A., Frid, V., 2005, Tensile Fracturing Rocks: Berlin, Springer, $579 \mathrm{pp}$.

Barton, C.C., LaPointe, P.R., 1995, Fractal Analysis of Scaling and Spatial Clustering of Fractures, in Barton, C., La Pointe, P.R. (eds.), Fractals in Earth Science: New York, Plenum Press, 141-178.

Barton, C.C., Larsen, E., 1985, Fractal geometry of two dimensional fracture networks at Yucca Mountain, south-western Nevada: Proceedings of International Symposium on Fundamentals of Rock Joints, Bjorkliden, Sweden, 77-84.

Bloomfield, K., 1975, A late Quaternary monogenetic volcano field in central Mexico: Geologische Rundschau, 64, 476-497.

Brogi, A., 2011, Variation in fracture patterns in damage zones related to strikeslip faults interfering with pre-existing fractures in sandstone (Calcione 
area, southern Tuscany, Italy): Journal of Structural Geology, 33, 644-661.

Brown, E.T., Werne, J.P., Lozano-García, M.S., Caballero-Miranda, M., OrtegaGuerrero, B., Cabral-Cano, E., Valero-Garcés, B.L., Schwalb, A., ArciniegaCeballos, A., 2012, Scientific Drilling in the Basin of Mexico to Evaluate Climate History, Hydrological Resources, and Seismic and Volcanic Hazards: Scientific Drilling, 14, 72-75, doi:10.2204/iodp.sd.14.12.2012.

Cadoux, A., Missenard, Y., Martínez-Serrano, R., Guillou, H., 2011, Trenchward Plio-Quaternary volcanism migration in the Trans-Mexican Volcanic Belt: the case of the Sierra Nevada range: Geological Magazine: 148(3), 492-506 doi:10.1017/S0016756810000993.

Caine, J.S., Evans, J.P., Forster, C.B., 1996, Fault zone architecture and permeability structure: Geology, 24(11), 1025-1028.

Cladouhos T.T., Marrett, R., 1996, Are fault growth and linkage models consistent with power-law distributions of fault lengths?: Journal of Structural Geology, 18, 281-293.

Cox, D.R., Lewis, P.A.W., 1966, The statistical analysis of series of events: London, Methuen's Monographs on Applied Probability and Statistics, 285 pp.

Davy, P., Sornette, A., Sornette, D., 1990, Some consequences of a proposed fractal nature of continental faulting: Nature, 348, 56-58.

Delgado-Granados, H., Nieto-Obregón, J., Silva-Romo, G., Mendoza- Rosales, C., Arellano-Gil, J., Lermo-Samaniego, J.F., Rodríguez- González., M., 1995, La Pera detachment fault system: active faulting south of Mexico City: geological evidence (II), en Reunión Anual de la Unión Geofísica Mexicana: Puerto Vallarta, Jalisco, GEOS, Resumen, 15(2), 64.

Ferrari, L., López-Martínez, M., Aguirre-Díaz, G., Carrasco-Núñez, G., 1999, Space-time patterns of Cenozoic arc volcanism in central Mexico: from the Sierra Madre Occidental to the Mexican Volcanic Belt: Geology, 27, 303-306.

Ferrari, L., Orozco-Esquivel, T., Manea, V., Manea, M., 2011, The dynamic history of the Trans-Mexican Volcanic Belt and the Mexico subduction zone: Tectonophysics, 522-523, 122-149.

Fries, C., 1956, Bosquejo Geológico de la región entre México, D.F. y Taxco Guerrero, en $20^{\circ}$ Congreso Geológico Internacional: México, D.F., Libreto de las excursiones, Excursión A-9 y C-12,11 pp.

Fries, C., 1960, Geología del Estado de Morelos y de partes adyacentes de México y Guerrero: Región central meridional de México: Boletín del Instituto de Geología, Universidad Nacional Autónoma de México, 60, 234 pp.

Gale, J.F.W., Laubach, S.E., Olson, J.E., Eichhubl, P., Fall, A., 2014, Natural fractures in shale. A review and new observations: American Association of Petroleum Geologists Bulletin, 98(11), 2165-2216.

García-Palomo, A., Macías, J. L., Garduño, V.H., 2000, Miocene to Recent structural evolution of the Nevado de Toluca volcano region, Central Mexico: Tectonophysics, 318, 281-302.

García-Palomo, A., Carlos-Valerio, V., López-Miguel, C., Galván-García, A., Concha-Dimas A., 2006, Landslide inventory map of Guadalupe Range, north of the Mexico Basin: Boletín de la Sociedad Geológica Mexicana, Número especial de Geología Urbana, LVIII(2), 195-204.

García-Palomo, A., Zamorano, J.J., López-Miguel, C., Galván-García, A., CarlosValerio, V., Ortega, R., Macías, J.L., 2008, El arreglo morfoestructural de la Sierra de Las Cruces, México central: Revista Mexicana de Ciencias Geológicas, 25(1), 158-178.

Gillespie, P.A., Howard, C.B., Wash J.J., Watterson J., 1993, Measurement and characterization of spatial distributions of fractures: Tectonophysics, 226, 113-141.

Gómez-Tuena A., Carrasco-Núñez, G., 2000, Cerro Grande volcano: the evolution of a Miocene stratocone in the early Trans-Mexican Volcanic Belt: Tectonophysics, 318, 249-280.

González-Torres E. A., Morán-Zenteno D. J., Mori, L., Martiny B. M., 2015, Revisión de los últimos eventos magmáticos del Cenozoico del sector norte-central de la Sierra Madre del Sur y su posible conexión con el subsuelo profundo de la Cuenca de México: Boletín de la Sociedad Geológica Mexicana, 67, 285-297.

Grassberger, P., Procaccia, I., 1983, Characterization of strange attractors: Physical Review Letters, 50, 346-349.

Griffith, A.A., 1920, The phenomena of rupture and flow in solids: Philosophical Transactions of the Royal Society of London, Series A, 221, 163-198.

Guilbaud, M.-N., Siebe, C., Agustín-Flores, J., 2009, Eruptive style of the young high-Mg basaltic-andesite Pelagatos scoria cone southeast of México City:
Bulletin of Volcanology, 71, 859-880.

Hooker, J.N., Laubach, S.E., Marrett, R., 2013, Fracture-aperture sized frequency, spatial distribution, and growth processes in strata-bounded and nonstratabounded fractures, Cambrian Meson Group, NW Argentina: Journal of Structural Geology, 54, 54-71.

Hooker, J.N., Laubach, S.E., Marrett, R., 2018, Microfracture spacing distributions and the evolution of fracture patterns in sandstones: Journal of Structural Geology, 108, 66-79.

Irwin, G.R., 1960, Fracture Mechanics, in Structural Mechanics, Proceedings of the First Symposium on Naval Structural Mechanics: Oxford, Pergamon Press, 557-594.

Jafari, A., Babadagli, T., 2008, A sensitivity analysis for effective parameters on fracture network permeability, in Society of Petroleum Engineers, Western Regional and Pacific Section of the American Association of Petroleum Geologists Joint Meeting: Bakersfield, California, United States of America, Paper SPE 113618, 17 pp.

Jafari, A., Babadagli, T., 2009, A sensitivity analysis for effective parameters on 2-D fracture network permeability: SPE Reservoir Evaluation and Engineering, 12(3), 455-469.

Jafari, A., Babadagli, T., 2010, Practical estimation of equivalent fracture network permeability of geothermal reservoirs, in 2010 World Geothermal Congress: Bali, Indonesia, Proceedings, 10.

Korvin, G., 1992, Fractal Models in the Earth Sciences: Amsterdam, Elsevier, $381 \mathrm{pp}$.

Laubach, S.E., Ward, M.E., 2006, Diagenesis in porosity evolution of openingmode fractures, Middle Triassic to Lower Jurassic La Boca Formation, NE Mexico: Tectonophysics, 419, 75-97.

Laubach, S.E., Lamarche, J., Gauthier, B.D.M., Dunne, W.M., Sanderson, D.J., 2018, Spatial arrangement of faults and opening-mode fractures: Journal of Structural Geology, 108, 2-15.

Lenhardt, N., Böhnel, H., Wemmer, K., Torres-Alvarado, I. S., Hornung, J., Hinderer, M., 2010, Petrology, magnetostratigraphy and geochronology of the Miocene volcaniclastic Tepoztlán Formation: implications for the initiation of the Transmexican Volcanic Belt (Central Mexico): Bulletin of Volcanology, 72, 817-832.

Lermo, J.F., Santoyo, M.A., Jaimes, M.A., Antayhua, Y., Chavacán, M., 2016, Local Earthquakes of México Basin in Mexico City: k, Q, Source Spectra, and Stress Drop: Bulletin of the Seismological Society of America, 106(4), 1423-1437.

Lezama-Campos, J.L., Morales-Casique, E., Castrejón-Pineda, R., Arce, J.L., Escolero, O.A., 2016, Interpretación del registro geofísico del pozo profundo San Lorenzo Tezonco y su correlación litológica en la cuenca de México: Revista Mexicana de Ciencias Geológicas, 33(2), 198-208.

Li, J.Z., Laubach, S.E., Gale, J.F.W., Marrett, R., 2018, Quantifying opening-mode fracture spatial organization in horizontal wellbore image logs, core and outcrop: Application to Upper Cretaceous Frontier Formation tight gas sandstones, USA: Journal of Structural Geology. 108, 137-156.

Lozano-Barraza, L., 1968, Geología de la Sierra de Guadalupe, México, D.F.: Instituto Politécnico Nacional, tesis de licenciatura, $39 \mathrm{pp}$.

Lozano-García, M.S., Ortega-Guerrero, B., 1998, Late Quaternary environmental changes of the central part of the Basin of Mexico; correlation between Texcoco and Chalco basins: Review of Palaeobotany and Palynology, 99, 77-93.

Lozano-García, M.S., Ortega-Guerrero, B., Caballero-Miranda, M., UrrutiaFucugauchi, J., 1993, Late Pleistocene and Holocene paleoenvironments of Chalco Lake, central Mexico: Quaternary Research, 40, 332-342.

Lozano-García, S., Brown, E.T., Ortega-Guerrero, B., Caballero, M., Werne, J., Fawcett, P.J., Schwalb, A., Valero-Garcés, B., Schnurrenberger, D., O'Grady, R., Stockhecke, M., Steinman, B., Cabral-Cano, E., Caballero, C., Sosa-Nájera, S., Soler, A.M., Pérez, L., Noren, A., Myrbo, A., Bücker, M., Wattrus, N., Arciniega, A., Wonik, T., Watt, S., Kumar, D., Acosta, C., Martínez, I., Cosío, R., Ferland, T., Vergara-Huerta, F., 2017, Perforación profunda en el lago de Chalco: reporte técnico: Boletín de la Sociedad Geológica Mexicana, 69(2), 299-311.

Macías, J.L., Arce, J.L., García-Tenorio, F., Layer, P.W., Rueda, H., Reyes-Agustín, G., López-Pizaña, F., Avellán, D., 2012, Geology and geochronology of Tlaloc, Telapón, Iztaccíhuatl, and Popocatépetl volcanoes, Sierra Nevada, central Mexico, in Aranda-Gómez, J.J., Tolson, G., Molina-Garza, R.S. (eds.), The Southern Cordillera and Beyond: Geological Society of America 
Field Guide, 25, 163-193.

Mandelbrot, B.B., 1967, How long is the coast of Britain? Statistical self-similarity and fractional dimension: Science, 156, 636-638.

Manea, V.C., Manea, M., Ferrari, L., 2013, A geodynamical perspective on the subduction of Cocos and Rivera plates beneath Mexico and Central America: Tectonophysics, 609, 56-81.

Márquez, A., Oyarzu, R., Doblas, M., Verma, S.P., 1999, Alkaline (oceanic island basalt type) and calc-alkaline volcanism in the Mexican Volcanic Belt: A case for plume-related magmatism and propagating rifting at an active margin?: Geology, 27, 51-54.

Martel, S.R., Pollar, D.D., 1989, Mechanics of slip and fracture along small faults and simple strike-slip fault zones in granitic rock: Journal of Geophysical Research, 94, 9417-9428

Marrett, R., Ortega, O.J., Kelsey, C.M., 1999, Extent of power-law scaling for natural fractures in rock: Geology, 27, 799-802.

Mauldon, M., Dunne, W., Rohrbaugh, M., 2001, Circular scanlines and circular windows: new tools for characterizing the geometry of fracture traces: Journal of Structural Geology, 23(2), 247-258.

Molnar, P., Sykes, L.R., 1969, Tectonics of the Caribbean and Middle American region from focal mechanisms and seismicity: Geological Society of America Bulletin, 80, 1639-1684.

Mooser, F., 1963, Historia tectónica de la Cuenca de México: Boletín de la Asociación Mexicana de Geólogos Petroleros, 15, 239-245.

Mooser, F., 1975, Historia geológica de la Cuenca de México, en Memoria de las obras de drenaje profundo del Distrito Federal: México, D.F., Departamento del Distrito Federal, 38 pp.

Morales-Casique, E., Escolero, O.A., Arce, J.L. 2014, Resultados del pozo San Lorenzo Tezonco y sus implicaciones en el entendimiento de la hidrogeología regional de la cuenca de México: Revista Mexicana de Ciencias Geológicas, 31(1), 64-75.

Morales-Casique, E., Escolero O.A., Arce J.L., 2015, Estimación de parámetros mediante inversión y análisis de las pérdidas hidráulicas lineales y nolineales durante el desarrollo y aforo del pozo San Lorenzo Tezonco: Boletín de la Sociedad Geológica Mexicana, 67(2), 203-214.

Morales-Casique, E., Arce-Saldaña J.L., Escolero-Fuentes O., Lezama-Campos J.L., 2016, Análisis de la estratigrafía y las características hidrogeológicas de los estratos profundos que conforman el subsuelo de la Cuenca de México a partir de la perforación de dos pozos profundos, uno a $2000 \mathrm{~m}$ y otro a 1570 m denominados Agrícola Oriental 2B y 2 C respectivamente: Reporte técnico para el Sistema de Aguas de la Ciudad de México, Gobierno de la Ciudad de México, 178 pp.

Morales-Casique, E., Arce-Saldaña J.L., Escolero O.A., Lezama-Campos, J.L., 2017, Analizar la estratigrafía y las características hidrogeológicas de los estratos profundos que conforman el subsuelo de la Cuenca de México a partir de la perforación a $2000 \mathrm{~m}$ del pozo profundo denominado "Santa Catarina 3": Reporte técnico para el Sistema de Aguas de la Ciudad de México, Gobierno de la Ciudad de México, 113 pp.

Moreno-Sánchez, G., García-Cabrejo, O.J., 2006, Caracterización Cuantitativa de Patrones de Fracturamiento mediante Ventanas Circulares y Análisis Fractal: Geología Colombiana, 31, 73-104.

Nieto-Samaniego, A.F., Alaniz-Álvarez, S., Tolson, G., Xu, S., Pérez-Venzor, A., 2003, Estimación de densidades, distribuciones de longitud y longitud total de fracturas: un caso de estudio en la Falla de Los Planes, La Paz, B.C.S.: Boletín de la Sociedad Geológica Mexicana, LVI(1), 1-9.

Nieto-Samaniego, A.F., Alaniz-Álvarez, S., Tolson, G., Oleschko, K., Korvin. G., Xu, S.S., Pérez- Venzor, J.A., 2005, Spatial Distribution, Scaling and Self-similar Behavior of Fracture Arrays in the Los Planes Fault, Baja California Sur, México: Pure Applied Geophysics, 162(2005), 805-826.

Norini, G., Groppelli, G., Lagmay, A.M.F., Capra, L., 2006, Recent left oblique slip faulting in the central eastern Trans-Mexican Volcanic Belt: Seismic hazard and geodynamic implications: Tectonics, 25(4), TC4012, 1-21, doi: 10.1029/2005TC001877.

Osete, M.L., Ruíz-Martínez, V.C., Caballero, M.C., Galindo, C., UrrutiaFucugauchi, J., Tarling, H.D., 2000, Southward migration of continental volcanic activity in the Sierra de Las Cruces, Mexico: Paleomagnetic and radiometric evidence: Tectonophysics, 318, 201-215.
Pardo, M., Suárez, G., 1993, Steep subduction geometry of the Rivera plate beneath the Jalisco block in western Mexico: Geophysical Research Letters, 20, 2391-2394.

Pardo, M., Suárez, G., 1995, Shape of the subducted Rivera and Cocos plates in southern Mexico: Seismic and tectonic implications: Journal of Geophysical Research, 100(87), 12357-12373.

Passchier, C.W., Trouw R.A.J., 1996, Microtectonics: Berlin, Springer Verlag, 366 pp.

PEMEX (Petróleos Mexicanos), SACMEX (Sistema de Aguas de la Ciudad de México), 2016a, Informe final de perforación del pozo Agrícola Oriental 2B: Ciudad de México, México, Equipo PM-1282, Reporte técnico, 52 pp.

PEMEX (Petróleos Mexicanos), SACMEX (Sistema de Aguas de la Ciudad de México), 2016b, Informe final de perforación del pozo Agrícola Oriental 2C: Ciudad de México, México, Equipo PM-1282, Reporte técnico, 40 pp.

Pérez-Cruz, G.A., 1988, Estudio sismológico de reflexión del subsuelo de la ciudad de México: México, D.F., Universidad Nacional Autónoma de México, tesis de maestría, 83 pp.

Ramsay, J.G., 1967, Folding and Fracturing of Rocks: New York, McGrawHill, 568 pp.

Ramsay, J.G., Huber, M.I., 1987, The Techniques of Modern Structural Geology, Vol. 2. Folds and Fractures: London, Academic Press, 391 pp.

Riley, P., Tikoff, B., Murray, A.B., 2011, Quantification of fracture networks in non-layered, massive rock using synthetic and natural data sets: Tectonophysics, 505, 44-56.

Rosenstein, M.T., Collins, J.J., De Luca, C.J., 1993, A practical method for calculating largest Lyapunov exponents from small data sets: Physica, $\mathrm{D}, 65,117-134$.

Siebe, C., 2000, Age and archaelogical implications of Xitle volcano, southwestern Basin of Mexico City: Journal of Volcanology and Geothermal Research, 104, 45-64.

Siebe, C., Arana-Salinas, L., Abrams, M., 2005, Geology and radiocarbon ages of Tláloc, Tlacotenco, Cuauhtzin, Hijo del Cuauhtzin, Teuhtli, and Ocusacayo monogenetic volcanoes in the central part of the Sierra Chichinautzin, México: Journal of Volcanology and Geothermal Research, 141, 225-243.

Stoiber, R.E., Carr, M.J., 1973, Quaternary volcanic and tectonic segmentation of Central America: Bulletin of Volcanology, 37, 304-325.

Sunye-Puchol, I., Lacan, P., Ontuño, M., Villamor, P., Audin, L., Zúñiga, F.R., Langridge, R.M., Aguirre-Díaz G. de J., Lawton, T.F., 2015, La falla San Mateo: nuevas evidencias paleosismológicas de fallamiento activo en el graben de Acambay, México: Revista Mexicana de Ciencias Geológicas, 32(3), 361-376.

Suter, M., López-Martínez, M., Quintero-Legorreta, O., Carrillo-Martínez, M., 2001, Quaternary intra-arc extension in the central Trans-Mexican volcanic belt: Geological Society of America Bulletin, 113(6), 693-703.

Turcotte, D., 1992, Fractals and chaos in Geology and Geophysics 1 Edition: Cambridge, Cambridge University Press, $221 \mathrm{pp}$.

Vásquez-Serrano, A., 2013, Análisis fractal de fracturas geológicas en un análogo expuesto de yacimientos carbonatados naturalmente fracturados y su aplicación en modelos de flujo de fluidos: México, D. F., Universidad Nacional Autónoma de México, tesis de maestría, 153 pp.

Velde, B., Dubois, J., Touchard, G., Badri, A., 1990, Fractal analysis of fractures in rocks: The Cantor's dust method: Tectonophysics, 179, 345-352.

Wallace, P., Carmichael, I.S.E., 1999, Quaternary volcanism near the Valley of Mexico: implications for subduction zone magmatism and the effects of crustal thickness variations on primitive magma compositions: Contributions to Mineralogy and Petrology, 135, 291-314.

Manuscrito recibido: mayo 7, 2018

Manuscrito corregido recibido: septiembre 6, 2018

Manuscrito aceptado: octubre 8, 2018 